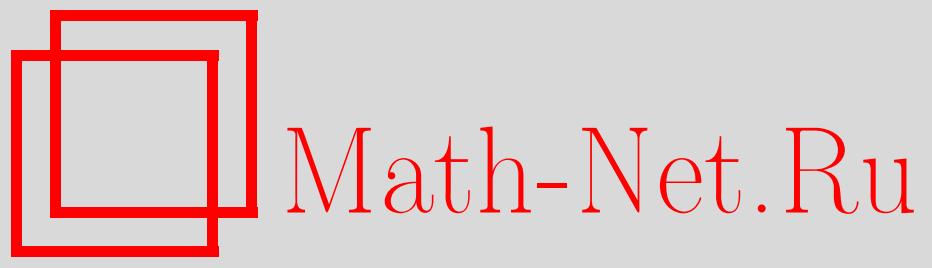

Л. Ю. Вострикова, Максимизация ожидаемой полезности для экспоненциальных моделей Леви с опционом и информационные процессы, Теория вероятн. и ее примен., 2016, том 61, выпуск 1, 26-52

DOI: https://doi.org/10.4213/tvp5042

Использование Общероссийского математического портала Math-Net.Ru подразумевает, что вы прочитали и согласны с пользовательским соглашением http://www . mathnet.ru/rus/agreement

Параметры загрузки:

IP : 54.198 .55 .26

26 апреля 2023 г., 04:44:16

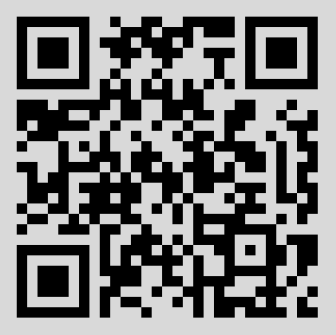




\title{
МАКСИМИЗАЦИЯ ОЖИДАЕМОЙ ПОЛЕЗНОСТИ ДЛЯ ЭКСПОНЕНЦИАЛЬНЫХ МОДЕЛЕЙ ЛЕВИ С ОПЦИОНОМ И ИНФОРМАЦИОННЫЕ ПРОЦЕССЫ ${ }^{1)}$
}

\begin{abstract}
Рассматривается задача максимизации ожидаемой полезности для экспоненциальных моделей Леви и полезностей типа HARA для случая, когда в портфеле ценных бумаг находится неликвидный актив. Этот неликвидный актив представлен опционом Европейского типа на второй актив, зависящий от первого ликвидного актива. При некоторых предположениях на процессы Леви, входящие в модель, даются выражения для информационных процессов, фигурирующих в формуле для максимальной ожидаемой полезности. В качестве примеров, рассматриваются модели Блэка и Шоулса с коррелированными броуновскими движениями, а также модели Блэка и Шоулса со скачками, представленными пуассоновским процессом.

Ключевые слова и фразы: максимизация ожидаемой полезности, экспоненциальная модель Леви, мартингальная мера, минимальная по $f$-дивергенции мера, дуальный метод, энтропия, информация Кульбака-Лейблера, информационные процессы.
\end{abstract}

1. Введение. Процессы Леви используются в финансовой математике в течение длительного времени. Эти модели включают в себя ряд популярных моделей со скачками, таких как обобщенные гиперболические модели и модели Variance-Gamma. Использование таких процессов для моделирования приводит к отличному соответствию как с данными о логарифме цены, отвечающими частым наблюдениям по одному фиксированному дню, так и с данными, отвечающими каждодневным наблюдениям. Класс процессов Леви достаточно широк и позволяет моделировать процессы с конечной и бесконечной вариациями, а также и процессы с конечной и бесконечной активностями. Процессы Леви не только хорошо описывают реальные данные, но и относительно просты для математических исследований (см. [8], [9] и указанные в этих работах ссылки).

*LAREMA, Département de Mathématiques, Université d'angers, 2 Bd. Lavoisier49045, Angers Cedex 01; e-mail: lioudmila.vostrikova@univ-angers.fr

1) Настоящая работа частично финансировалась грантом ANR-09-BLAN-0084-01 кафедры математики Университета г. Анжэ, Франция. 
Обозначим $X=\left(X_{t}\right)_{t \geqslant 0}$ процесс Леви размерности $d, d \geqslant 1$, с триплетом характеристик $(b, c, K)$, где $b \in \mathbf{R}^{d}$ - параметр сноса, $c$ является $(d \times d)$-матрицей, характеризующей вариацию непрерывной мартингальной компоненты процесса $X$, а $K$ является мерой Леви этого процесса и удовлетворяет условию

$$
\int_{\mathbf{R}^{d}}\left(\|x\|^{2} \wedge 1\right) K(d x)<\infty
$$

Как известно, закон распределения такого процесса полностью определяется его одномерным распределением, а характеристическая функция $X_{t}={ }^{\top}\left(X_{t}^{1}, X_{t}^{2}, \ldots, X_{t}^{d}\right)$, отвечающая параметру $\lambda \in \mathbf{R}^{d}$, равна

$$
\phi_{X_{t}}(\lambda)=\exp \{t \psi(\lambda)\}
$$

и, в свою очередь, характеристическая экспонента процесса $X$ задается формулой

$$
\psi(\lambda)=\exp \left\{i^{\top} \lambda b-\frac{1}{2}{ }^{\top} \lambda c \lambda+\int_{\mathbf{R}^{d}}\left(e^{i^{\top} \lambda x}-1-i^{\top} \lambda l(x)\right) K(d x)\right\}
$$

с урезающей функцией $l$. Как правило, урезающая функция $l: \mathbf{R}^{d} \rightarrow \mathbf{R}^{d}$ является ограниченной функцией с компактным носителем такой, что $l(x)=x$ в окрестности нуля. При стандартном выборе $l, l(x)=x \mathbf{1}_{\{\|x\| \leqslant 1\}}$, где $\mathbf{1}_{\{\cdot\}}$ обозначает индикатор, а $\|\cdot\|$ - эвклидова норма в $\mathbf{R}^{d}$ (более подробно о процессах Леви см. [2], [19]).

Как обычно, мы будем моделировать цену акций с помощью стохастической экспоненты от уже выбранного процесса Леви $X$, а именно возьмем $S=\left(S_{t}\right)_{t \geqslant 0}$ с

$$
S_{t}={ }^{\top}\left(\mathscr{E}\left(X^{1}\right)_{t}, \mathscr{E}\left(X^{2}\right)_{t}, \ldots, \mathscr{E}\left(X^{d}\right)_{t}\right),
$$

где $\mathscr{E}(\cdot)$ обозначает экспоненту Долеан-Дадэ, а $X^{i}, 1 \leqslant i \leqslant d$, являются компонентами процесса $X$. Напомним, что для каждого одномерного семимартингала $X^{i}$

$$
\mathscr{E}\left(X^{i}\right)_{t}=\exp \left\{X_{t}^{i}-\frac{1}{2}\left\langle X^{i, c}\right\rangle_{t}\right\} \prod_{0 \leqslant s \leqslant t} \exp \left\{-\Delta X_{s}^{i}\right\}\left(1+\Delta X_{s}^{i}\right) .
$$

В предшествующем равенстве $\left\langle X^{i, c}\right\rangle$ обозначает квадратическую вариацию непрерывной мартингальной части процесса $X^{i}$, а $\Delta X^{i}$ обозначает скачки процесса $X^{i}$ (см. подробнее [16]).

Задача максимизации ожидаемой полезности для экспоненциальных моделей Леви в одномерном случае расматривалась во многих работах (см., например, [6], [7] и указанные в этих работах ссылки). Однако же случай максимизации полезности, когда в портфеле ценных бумаг находится неликвидный актив в виде опциона, не был полностью изучен. 
Некоторые результаты в этом направлении можно найти в [3], [4], [5], [13], [17], [18]. Формулы для максимальной ожидаемой полезности были получены в случае, когда в модели фигурируют только броуновские движения, а неполнота рынка порождается активом, управление которым невозможно (см. [13], [17], [18]). Формулы для максимальной ожидаемой полезности в случае полных рынков были получены в [1]. Однако же случай, когда модели Леви ликвидного и неликвидного активов являются, с одной стороны, зависимыми, а с другой стороны, содержат скачкообразную компоненту, до сих пор не рассматривался.

Чтобы моделировать зависимые активы с помощью процессов Леви, введем два независимых процесса Леви размерности $d$, обозначенные $X^{(1)}$ и $X^{(2)}$ и характеризуемые триплетами $\left(b_{1}, c_{1}, K_{1}\right)$ и $\left(b_{2}, c_{2}, K_{2}\right)$ соответственно, где $K_{1}$ и $K_{2}$ удовлетворяют условию типа (1.1). Для двух обратимых матриц $\rho_{1}$ и $\rho_{2}$ с действительными компонентами, введем процесс $X=\left(X_{t}\right)_{0 \leqslant t \leqslant T}$, являющийся линейной комбинацией процессов $X^{(1)}$ и $X^{(2)}$, а именно

$$
X_{t}=\rho_{1} X_{t}^{(1)}+\rho_{2} X_{t}^{(2)} .
$$

Будем предполагать, что цены акций моделируются процессами $S^{(1)}=$ $\left(S_{t}^{(1)}\right)_{0 \leqslant t \leqslant T}$ и $S^{(2)}=\left(S_{t}^{(2)}\right)_{0 \leqslant t \leqslant T^{\prime}}$ с $T^{\prime}>T$ и

$$
S_{t}^{(1)}=^{\top}\left(\mathscr{E}\left(X^{1}\right)_{t}, \mathscr{E}\left(X^{2}\right)_{t}, \ldots, \mathscr{E}\left(X^{d}\right)_{t}\right)
$$

и

$$
S_{t}^{(2)}=^{\top}\left(\mathscr{E}\left(X^{(2), 1}\right)_{t}, \mathscr{E}\left(X^{(2), 2}\right)_{t}, \ldots, \mathscr{E}\left(X^{(2), d}\right)_{t}\right) .
$$

Чтобы обеспечить положительность каждой компоненты процессов $S^{(1)}$ и $S^{(2)}$ предположим, что для $1 \leqslant i \leqslant d$, скачки процессов $X^{i}$ and $X^{(2), i}$ удовлетворяют условию: $\Delta X_{t}^{i}>-1, \Delta X_{t}^{(2), i}>-1$. Не теряя общности, будем считать, что процентная ставка банковского счета $r$ равна нулю.

В описанной выше ситуации, инвестор, имеющий два актива, $S^{(1)}$ и $S^{(2)}$, может управлять первым активом $S^{(1)}$ с временем жизни $T$, а второй актив, $S^{(2)}$, с временем жизни $T^{\prime}>T$, для управления ему недоступен из-за отсутствия ликвидности или из-за административных ограничений. В то же самое время, у инвестора имеется опцион европейского типа $g\left(X_{T^{\prime}}^{(2)}\right)$ на второй актив, где $g$ является неотрицательной действительной борелевской функцией в $\mathbf{R}^{d}$, который он может продать. В такой ситуации, чтобы принять решение о продаже, для инвестора важно оценить максимальную ожидаемую полезность портфеля, содержащего опцион.

Обозначим $\Pi(\mathbb{F})$ множество самофинансируемых допустимых стратегий по отношению к фильтрации $\mathbb{F}$, порожденной процессом $X$. Тогда, для функции полезности $u$ и начального капитала $x_{0}$, максимальная ожидаемая полезность $U_{T}\left(x_{0}, 0\right)$, связанная только лишь с первым активом 
$S^{(1)}$, определяется выражением

$$
U_{T}\left(x_{0}, 0\right)=\sup _{\phi \in \Pi(\mathbb{F})} \mathbf{E}\left[u\left(x_{0}+\int_{0}^{T} \phi_{s} \cdot d S_{s}^{(1)}\right)\right],
$$

а если добавлен опцион, то максимальная ожидаемая полезность становится равной

$$
U_{T}\left(x_{0}, g\right)=\sup _{\phi \in \Pi(\mathbb{G})} \mathbf{E}\left[u\left(x_{0}+\int_{0}^{T} \phi_{s} \cdot d S_{s}^{(1)}+g\left(X_{T^{\prime}}^{(2)}\right)\right)\right],
$$

где $\Pi(\mathbb{G})$ - множество самофинансируемых допустимых стратегий по отношению к расширенной фильтрации $\mathbb{G}=\left(\mathscr{G}_{t}\right)_{0 \leqslant t \leqslant T}$ такой, что для $t \in 0 \leqslant t<T$

$$
\mathscr{G}_{t}=\bigcap_{s>t} \mathscr{F}_{s} \otimes \sigma\left(X_{T^{\prime}}^{(2)}\right) \quad \text { и } \quad \mathscr{G}_{T}=\mathscr{F}_{T} \otimes \sigma\left(X_{T^{\prime}}^{(2)}\right) .
$$

Указанный выше подход совпадает с так называемой максимизацией ожидаемой полезности при наличии искажения. В случае экспоненциальных моделей Леви, роль искажения играет $\delta=X_{T^{\prime}}^{(2)}-X_{T}^{(2)}$, при этом информация, содержащаяся в $\mathscr{G}_{T}$, совпадает с информацией, содержащейся в $\sigma$-алгебре $\mathscr{F}_{T}^{(1)} \otimes \mathscr{F}_{T}^{(2)} \vee \sigma\left(X_{T^{\prime}}^{(2)}-X_{T}^{(2)}\right)$, т.е. с прогрессивной фильтрацией в момент времени $T$, расширенной $\sigma$-алгеброй, порожденной искажением.

В данной работе рассматривается задача нахождения максимальной ожидаемой полезности для неполных рынков и моделей, представленных экспоненциальными моделями Леви, являющимися зависимыми. Как правило, в задаче нахождения максимальной ожидаемой полезности, используются так называемые HARA-полезности. Kaк известно, HARAполезности можно определить через коэффициент absolute risk aversion:

$$
R(x)=-\frac{u^{\prime \prime}(x)}{u^{\prime}(x)} .
$$

В этом случае,

$$
R(x)=\frac{1}{A+B x},
$$

где $A$ и $B$ - положительные константы. Решения указанного дифференциального уравнения для $u$ известны и представляют собой логарифмическую, степенную и экспоненциальную полезности

$$
\begin{aligned}
& u(x)=\ln x, \quad \text { где } x \in \mathbf{R}^{+, *}, \\
& u(x)=\frac{x^{p}}{p}, \quad \text { где } x \in \mathbf{R}^{+, *} \text { и } p \in(-\infty, 0) \cup(0,1), \\
& u(x)=1-e^{-\gamma x}, \quad \text { где } x \in \mathbf{R} \text { и } \gamma>0 .
\end{aligned}
$$


Задача максимизации ожидаемой полезности портфеля ценных бумаг с опционом в случае, когда $X$ и $X^{(2)}$ являются семимартингалами, рассматривалась в работе [10]. Используемый метод был основан на расширении фильтрации, последующем рассмотрении соответствующей условной задачи, при условии $X_{T^{\prime}}^{(2)}$ и, наконец, использовании дуального метода. В упомянутом дуальном методе, нахождение максимальной ожидаемой полезности сводится к поиску локально эквивалентной минимальной мартингальной меры, отвечающей $f$-дивергенции. Напомним, что $f$-дивергенция связана с полезностью $u$ преобразованием ЛежандраФенхеля по формуле

$$
f(x)=\sup _{y \in \mathbf{R}}(u(y)-x y) .
$$

Как известно, если $u$ - логарифмическая функция, то

$$
f(x)=-\ln x-1,
$$

если $u$ - степенная, то

$$
f(x)=\frac{1-p}{p} x^{p /(p-1)},
$$

и если $u$ - экспоненциальная, то

$$
f(x)=1-\frac{x}{\gamma}(1+\ln \gamma)+\frac{1}{\gamma} x \ln x .
$$

В разд. 2 мы приводим, для удобства читателей, некоторые результаты о максимизации ожидаемой полезности с опционом, отвечающие семимартингальным моделям. Основными результатами этого раздела являются формулы для максимальной ожидаемой полезности. Приведенные формулы содержат соответствующие информационные количества, такие как энтропия, информация Кульбака-Лейблера и интегралы типа интегралов Хеллингера. В свою очередь, эти информационные количества могут быть легко выражены через соответствующие информационные процессы.

В разд. 3 рассматриваются экспоненциальные модели Леви. А именно, проверяются условия, необходимые для применения результатов разд. 2, и даются выражения для параметров Гирсанова условной, мартингальной, минимальной по рассматриваемой $f$-дивергенции меры. Полученные параметры позволяют написать выражения для соответствующих информационных процессов, и затем, применить результаты разд. 2.

В разд. 4 даются выражения для информационных количеств, отвечающих моделям Блэка и Шоулса с коррелированными броуновскими движениями.

В разд. 5 рассматриваются модели Блэка и Шоулса с коррелированными броуновскими движениями и скачками, представленными пуассоновским процессом. 


\section{2. Некоторые известные результаты о максимизации ожи-} даемой полезности для экспоненциальных семимартингальных моделей.

2.1. Вероятностная модель и предположения. Пусть наблюдается процесс $X=\left(X_{t}\right)_{0 \leqslant t \leqslant T}$, заданный на каноническом вероятностном пространстве $(\Omega, \mathscr{F}, P)$ с фильтрацией $\mathbb{F}=\left(\mathscr{F}_{t}\right)_{0 \leqslant t \leqslant T}$, удовлетворяющей стандартным условиям. Введенный процесс представляет собой вектор стохастических логарифмов $d$-мерного ликвидного актива $S^{(1)}=\left(S_{t}^{(1)}\right)_{0 \leqslant t \leqslant T}$ такого, что

$$
S_{t}^{(1)}={ }^{\top}\left(\mathscr{E}\left(X^{1}\right)_{t}, \mathscr{E}\left(X^{2}\right)_{t}, \ldots, \mathscr{E}\left(X^{d}\right)_{t}\right)
$$

Кроме того, имеется $d$-мерный семимартингал $X^{(2)}$, представляющий собой стохастические логарифмы второго неликвидного актива. Этот неликвидный актив включен в портфель в виде опциона Европейского типа, а именно $g\left(X_{T^{\prime}}^{(2)}\right)$, где $g$ - положительная измеримая функция в пространстве $\mathbf{R}^{d}$ и $T^{\prime}>T$. Для получения результатов по максимизации ожидаемой полезности, давайте введем пространство $\left(\Omega \times \mathbf{R}^{d}, \mathscr{F} \otimes\right.$ $\left.\sigma\left(X_{T^{\prime}}^{(2)}\right), P \times \alpha\right)$, где $P$ — так называемый наблюдаемый закон распределения процесса $X$, а $\alpha$ - наблюдаемый закон распределения $X_{T^{\prime}}^{(2)}$. Рассматриваемая нами на этом пространстве фильтрация будет расширенной, а именно $\mathbb{G}=\left(\mathscr{G}_{t}\right)_{0 \leqslant t \leqslant T}$, где

$$
\mathscr{G}_{t}=\bigcap_{s>t} \mathscr{F}_{s} \otimes \sigma\left(X_{T^{\prime}}^{(2)}\right) \quad \text { и } \quad \mathscr{G}_{T}=\mathscr{F}_{T} \otimes \sigma\left(X_{T^{\prime}}^{(2)}\right) .
$$

Заметим, что $X$ будет семимартингалом на введенном пространстве с расширенной фильтацией $\mathbb{G}$, поскольку процессы $X$ и $X^{(2)}$ являются независимыми по мере $P \times \alpha$.

Теперь давайте обозначим $\mathbb{P}$ закон распределения пары $\left(X, X_{T^{\prime}}^{(2)}\right)$ и рассмотрим условные регулярные законы распределения $P^{v}$ процесса $X$ при условии $\left\{X_{T^{\prime}}^{(2)}=v\right\}$, более точно для $A \in \mathscr{F}$ и $v \in \mathbf{R}^{d}$

$$
P^{v}(A)=\mathbb{P}\left(A \mid X_{T^{\prime}}^{(2)}=v\right) .
$$

Введем условие, нужное для того, чтобы $X$ остался семимартингалом по мере $P^{v}$.

П р е д п о ло ж е н и е 1 . Для каждого $v \in \mathbf{R}^{d}$ вероятностная мера $P^{v}$ локально абсолютно непрерьвна по отношению $\kappa$ мере $P$, т.е.

$$
P^{v} \stackrel{\text { loc }}{\ll} P \text {. }
$$

Если предположение 1 выполнено, то согласно [15] и [16], процесс $X$ будет семимартингалом не только по мере $P$, но также и по мере $P^{v}$, 
$v \in \mathbf{R}^{d}$. Конечно, семимартингальные характеристики этого процесса изменятся в соответствии с теоремой 3.24 [16, с. 159].

Для $0 \leqslant t \leqslant T$ обозначим $P_{t}^{v}$ и $P_{t}$ сужения мер $P^{v}$ и $P$ на $\sigma$ алгебру $\mathscr{F}_{t}$. Чтобы избежать осложнений, связанных с измеримостью в стохастическом исчислении с процессами, зависящими от параметра $v$ (см. [20]), давайте рассмотрим опциональные версии процессов правдоподобия $\left(d P_{t}^{v} / d P_{t}\right)_{0 \leqslant t \leqslant T}$ по отношению к фильтрации $\mathbb{G}$. Для этого рассмотрим условное распределение случайной величины $X_{T^{\prime}}^{(2)}$ при условии $\mathscr{F}_{t}$, т.е.

$$
\alpha^{t}(\omega, d v)=\mathbb{P}\left(X_{T^{\prime}}^{(2)} \in d v \mid \mathscr{F}_{t}\right)(\omega) .
$$

П р е д по ло ж ен и е 2. Условное распределение случайной величинь $X_{T^{\prime}}^{(2)}$ при условии $\mathscr{F}_{t}$ абсолютно непрерывно по отношению $\kappa$ ее закону распределения, т.е.

$$
\left.\left.\alpha^{t} \ll \alpha \quad \forall t \in\right] 0, T\right]
$$

Согласно лемме Жакода (см. [14]) предположение 2 обеспечивает существование опциональной версии процесса $\left(d \alpha^{t} / d \alpha\right)_{0 \leqslant t \leqslant T}$.

3 а м е ч а н и е 1 . Следует также заметить, что предположение 2 можно заменить предположением, что случайная величина $d P_{T}^{v} / d P_{T}$, рассматриваемая как функция $(\omega, v)$, является $\mathscr{F}_{T} \otimes \mathscr{B}\left(\mathbf{R}^{d}\right)$-измеримой. Действительно, в указанном случае, можно построить опциональную версию процесса, фигурирующего в предположении 2, используя результаты [20].

Как уже было сказано, следующим этапом, позволяющим получить формулы для максимальной ожидаемой полезности, является решение так называемой условной проблемы максимизации полезности, используя дуальный метод (см., например, [11]). Для этого обозначим с помощью $f$ дуальную, по отношению к $u$, функцию. Пусть $\mathscr{M}_{T}^{v}$ обозначает множество эквивалентных мартингальных мер, определенных на вероятностном пространстве $\left(\Omega, \mathscr{F}_{T}, P_{T}^{v}\right)$ для семимартингальной модели $S^{(1)}$, и пусть

$$
\mathscr{K}_{T}^{v}=\left\{Q_{T}^{v} \in \mathscr{M}_{T}^{v}: \mathbf{E}_{P^{v}}\left|f\left(\frac{d Q_{T}^{v}}{d P_{T}^{v}}\right)\right|<\infty\right\} .
$$

Напомним, что $Q_{T}^{v, *}$ является эквивалентной мартингальной мерой, минимизирующей $f$-дивергенцию, если

$$
\mathbf{E}_{P^{v}}\left[f\left(\frac{d Q_{T}^{v, *}}{d P_{T}^{v}}\right)\right]=\min _{Q_{T}^{v} \in \mathscr{K}_{T}^{v}} \mathbf{E}_{P^{v}}\left[f\left(\frac{d Q_{T}^{v}}{d P_{T}^{v}}\right)\right] .
$$

Чтобы воспользоваться дуальным методом, сделаем следующее предположение. 
П р е д п о ло ж е н и е 3. Для каждого $v \in \mathbf{R}^{d}$, существует эквивалентная мартингальная мера $Q_{T}^{v, *}$, минимизирующая $f$-дивергениию, принадлежащая классу $\mathscr{K}_{T}^{v}$ и такая, что случайная величина $z_{T}^{*}(v)=d Q_{T}^{v, *} / d P_{T}^{v}$, рассматриваемая как функция от $(\omega, v)$, является $\mathscr{F}_{T} \otimes \mathscr{B}\left(\mathbf{R}^{d}\right)$-измеримой, а случайная величина $\mathbf{E}_{P^{v}} f\left(z_{T}^{*}(v)\right)$ - интегрируемой (nо $v$ ) по мере $\alpha$.

2.2. Существование мартингальной меры, минимизирующей $f$-дивергенцию. Напомним общие результаты о существовании мартингальной меры, минимизирующей $f$-дивергенцию. Для этого мы обозначим $\mathbb{P}_{T}$ сужения меры $\mathbb{P}$ на $\sigma$-алгебру $\mathscr{G}_{T}$, и пусть $\mathscr{M}_{T}$ обозначает семейство эквивалентных мартингальных мер для семимартингала $\left(S_{t}^{(1)}\right)_{0 \leqslant t \leqslant T}$, рассматриваемого как отображение, заданное на вероятностном пространстве $\left(\Omega_{T}^{(1)} \times \mathbf{R}^{d}, \mathscr{F}^{(1)} \otimes \mathscr{B}\left(\mathbf{R}^{d}\right), \mathbb{P}_{T}\right)$ с фильтрацией $\mathbb{G}$. Обозначим

$$
\mathscr{K}_{T}=\left\{\mathbb{Q}_{T} \in \mathscr{M}_{T}\left|\mathbf{E}_{\mathbb{P}}\right| f\left(\frac{d \mathbb{Q}_{T}}{d \mathbb{P}_{T}}\right) \mid<\infty\right\} .
$$

Заметим, что $\mathscr{K}_{T} \neq \emptyset$. На самом деле, в качестве производной РадонаНикодима меры $\mathbb{Q}_{T}$ по отношению к мере $\mathbb{P}_{T}$, можно взять $z_{T}^{*}(v)$ из предположения 3 с заменой $v$ на $X_{T^{\prime}}^{(2)}$. Напомним, также, что мера $\mathbb{Q}_{T}^{*}$ является мартингальной мерой, минимизирующей $f$-дивергенцию, если

$$
\inf _{\mathbb{Q}_{T} \in \mathscr{K}_{T}} \mathbf{E}_{\mathbb{P}} f\left(\frac{d \mathbb{Q}_{T}}{d \mathbb{P}_{T}}\right)=\mathbf{E}_{\mathbb{P}} f\left(\frac{d \mathbb{Q}_{T}^{*}}{d \mathbb{P}_{T}}\right) .
$$

Теорема 1 (см. [10]). Если предположения 1, 2 и 3 вылолнень, то существует вероятностная мера $\mathbb{Q}_{T}^{*} \in \mathscr{K}_{T}$, являющаяся мартингальной мерой, минимизирующей $f$-дивергениию. Более того,

$$
U_{T}\left(x_{0}, g\right)=\int_{\mathbf{R}^{d}} \mathbf{E}_{P^{v}}\left[u\left(-f^{\prime}\left(\lambda_{g}(v) z_{T}^{*}(v)\right)\right)\right] d \alpha(v),
$$

где $\lambda_{g}(v)$ является решением уравнения

$$
-\mathbf{E}_{P^{v}}\left[f^{\prime}\left(\lambda_{g}(v) z_{T}^{*}(v)\right)\right]=x_{0}+g(v)
$$

2.3. Условные информационные количества и максимальная ожидаемая полезность. Предположим, что эквивалентная мартингальная мера, минимизирующая $f$-дивергенцию, существует и равна $Q_{T}^{v, *} \in \mathscr{K}_{T}^{v}$. Обозначим

$$
z_{T}^{*}(v)=\frac{Q_{T}^{v, *}}{P_{T}^{v}}, \quad p_{T}^{v}=\frac{d P_{T}^{v}}{d P_{T}} .
$$

Введем информационные количества, отвечающие мерам $P_{T}^{v}$ и $Q_{T}^{v, *}$, а именно энтропию меры $P_{T}^{v}$ по отношению к мере $Q_{T}^{v \text {,* }}$

$$
\mathbf{I}\left(P_{T}^{v} \mid Q_{T}^{v, *}\right)=-\mathbf{E}_{P^{v}}\left[\ln z_{T}^{*}(v)\right]=-\mathbf{E}_{P}\left[p_{T}^{v} \ln z_{T}^{*}(v)\right],
$$


затем, энтропию меры $Q_{T}^{v, *}$ по отношению к мере $P_{T}^{v}$, называемую также информацией Кульбака-Лейблера

$$
\mathbf{I}\left(Q_{T}^{v, *} \mid P_{T}^{v}\right)=\mathbf{E}_{P^{v}}\left[z_{T}^{*}(v) \ln z_{T}^{*}(v)\right]=\mathbf{E}_{P}\left[p_{T}^{v} z_{T}^{*}(v) \ln z_{T}^{*}(v)\right],
$$

и, наконец, интегралы типа интегралов Хеллингера

$$
\mathbf{H}_{T}^{(q), *}(v)=\mathbf{E}_{P^{v}}\left[\left(z_{T}^{*}(v)\right)^{q}\right]=\mathbf{E}_{P}\left[p_{T}^{v}\left(z_{T}^{*}(v)\right)^{q}\right],
$$

где $q=p /(p-1)$ и $p<1$.

Теперь дадим выражения для максимальной ожидаемой полезности, включающие в себя введенные выше энтропии и интегралы типа Хеллингера.

Теорема 2 (см. [10]). При выполнении предположений 1, 2 и 3, существует $\mathscr{B}\left(\mathbf{R}^{d}\right)$-измеримая версия введенных информачионньх количеств. Более того, максимальная ожидаемая полезность $U_{T}\left(x_{0}, g\right)$ равна

(i) в случае $u(x)=\ln x$,

$$
U_{T}\left(x_{0}, g\right)=\int_{\mathbf{R}^{d}}\left[\ln x_{0}+g(v)+\mathbf{I}\left(P_{T}^{v} \mid Q_{T}^{v, *}\right)\right] d \alpha(v) .
$$

(ii) в случае $u(x)=x^{p} / p$, где $p<1, p \neq 0$,

$$
U_{T}\left(x_{0}, g\right)=\frac{1}{p} \int_{\mathbf{R}^{d}}\left(x_{0}+g(v)\right)^{p}\left(\mathbf{H}_{T}^{(q), *}(v)\right)^{1-p} d \alpha(v) .
$$

(iii) в случае $u(x)=1-e^{-\gamma x}$, где $\gamma>0$,

$$
U_{T}\left(x_{0}, g\right)=1-\int_{\mathbf{R}^{d}} \exp \left\{-\left[\gamma\left(x_{0}+g(v)\right)+\mathbf{I}\left(Q_{T}^{v, *} \mid P_{T}^{v}\right)\right]\right\} d \alpha(v) .
$$

2.4. Условные информационные процессы и условные информационные количества. В этом разделе мы напомним, каким образом информационные количества связаны с информационными процессами. Чтобы упростить выражения, задающие информационные процессы, в этом разделе мы будем предполагать, что процесс $X$ является квазинепрерывным слева. Напомним, что $(P, \mathbb{F})$-семимартингал $X$ является квазинепрерывным слева, если для любого предсказуемого момента остановки $\tau$, скачок процесса $\Delta X_{\tau}=0$ ( $P$-a.s. $)$ на множестве $\{\tau<\infty\}$. Заметим, что поскольку $P^{v} \stackrel{\text { loc }}{\ll} P,\left(P^{v}, \mathbb{F}\right)$-семимартингал $X$ будет также квазинепрерывным слева.

Обозначим $\beta^{v, *}$ и $Y^{v, *}$ два $\left(P^{v}, \mathbb{F}\right)$-предсказуемых процесса, известных как параметры Гирсанова при замене меры $P^{v}$ на $Q^{v, *}$ таких, что для любого $t \geqslant 0$ и $P^{v}$-п.н.

$$
\int_{0}^{t} \int_{\mathbf{R}^{d}}\|l(x)\|\left|\left(Y_{s}^{v, *}(x)-1\right)\right| \nu^{v}(d s, d x)<\infty
$$


И

$$
\int_{0}^{t}\left\|c_{s} \beta_{s}^{v, *}\right\| d s<\infty, \quad \int_{0}^{t}{ }^{\top} \beta_{s}^{v, *} c_{s} \beta_{s}^{v, *} d s<\infty .
$$

В предыдущем выражении $\nu^{v}$ обозначает компенсатор меры скачков процесса $X, l$ является урезающей функцией, а $c$ служит для обозначения плотности вариации непрерывной мартингальной части процесса $X$ по мере Лебега.

В случае логарифмической полезности, соответствующим информационным количеством является энтропия $\mathbf{I}\left(P_{T}^{v} \mid Q_{t}^{v, *}\right)$, а соответствующий информационный процесс $\mathscr{I}^{*}(v)=\left(\mathscr{I}_{t}^{*}(v)\right)_{t \in[0, T]}$ задается формулой

$$
\begin{aligned}
\mathscr{I}_{t}^{*}(u)= & \frac{1}{2} \int_{0}^{t}{ }^{\top} \beta_{s}^{v, *} c_{s} \beta_{s}^{v, *} d s \\
& -\int_{0}^{t} \int_{\mathbf{R}^{d}}\left[\ln \left(Y_{s}^{v, *}(x)\right)-Y_{s}^{v, *}(x)+1\right] \nu^{v}(d s, d x) .
\end{aligned}
$$

Предложение 1. Предположим, что $Q_{T}^{v, *} \in \mathscr{K}_{T}^{v}$. Тогда coomветствующая энтропия конечна и

$$
\mathbf{I}\left(P_{T}^{v} \mid Q_{T}^{v, *}\right)=\mathbf{E}_{P^{v}} \mathscr{I}_{T}^{*}(v) .
$$

В случае экспоненциальной полезности, рассмотрим информацию Кульбака-Лейблера $\mathbf{I}\left(Q_{T}^{v, *} \mid P_{T}^{v}\right)$ и соответствующий информационный процесс Кульбака-Лейблера $I^{*}(v)=\left(I_{t}^{*}(v)\right)_{t \in[0, T]}$ такой, что

$$
\begin{aligned}
I_{t}^{*}(v)= & \frac{1}{2} \int_{0}^{t} \top \beta_{s}^{v, *} c_{s} \beta_{s}^{v, *} d s \\
& +\int_{0}^{t} \int_{\mathbf{R}^{d}}\left[Y_{s}^{v, *}(x) \ln \left(Y_{s}^{v, *}(x)\right)-Y_{s}^{v, *}(x)+1\right] \nu^{v}(d s, d x) .
\end{aligned}
$$

Предложение 2. Предположим, ито $Q_{T}^{v, *} \in \mathscr{K}_{T}^{v}$. Тогда соответствующая информачия Кульбака-Лейблера конечна и

$$
\mathbf{I}\left(Q_{T}^{v, *} \mid P_{T}^{v}\right)=\mathbf{E}_{P^{v}}\left[\int_{0}^{T} z_{s-}^{*}(v) d I_{s}^{*}(v)\right]=\mathbf{E}_{Q^{v, *}}\left(I_{T}^{*}(v)\right) .
$$

В случае степенной полезности рассмотрим интегралы типа Хеллингера

$$
\mathbf{H}_{T}^{(q), *}(v)=\mathbf{E}_{P^{v}}\left[\left(z_{T}^{*}(v)\right)^{q}\right]
$$

где $q=p /(p-1)<1$.

Отвечающие этим интегралам предсказуемые процессы, $h^{(q), *}(v)=$ $\left(h_{t}^{(q), *}(v)\right)_{t \in[0, T]}$, называемые процессами Хеллингера, определяются выражением

$$
\begin{aligned}
h_{t}^{(q), *}(v)= & \frac{1}{2} q(1-q) \int_{0}^{t} \mathrm{\top} \beta_{s}^{v, *} c_{s} \beta_{s}^{v, *} d s \\
& -\int_{0}^{t} \int_{\mathbf{R}^{d}}\left[\left(Y_{s}^{v, *}(x)\right)^{q}-q\left(Y_{s}^{v, *}(x)-1\right)-1\right] \nu^{v}(d s, d x) .
\end{aligned}
$$


Предложение 3. Предположим, что $Q_{T}^{v, *} \in \mathscr{K}_{T}^{v}$. Тогда соответствуюшие интеграль Хеллингера $\mathbf{H}_{T}^{(q), *}(v)$ конечны и

$$
\mathbf{H}_{T}^{(q), *}(v)=1-\mathbf{E}_{P^{v}}\left[\int_{0}^{T}\left(z_{s-}^{*}(v)\right)^{q} d h_{s}^{(q), *}(v)\right]
$$

или же, в терминах стохастической экспоненть,

$$
\mathbf{H}_{T}^{(q), *}(v)=\mathbf{E}_{R^{v}}\left[\mathscr{E}\left(-h^{(q), *}(v)\right)_{T}\right],
$$

где $R^{v}$ - некоторая локально абсолютно непрерьвная по отношению $\kappa P^{v}$ мера.

\section{3. Максимизация ожидаемой полезности для экспонен-} циальных моделей Леви с опционом. Напомним основные обозначения, необходимые для формулировки результатов о максимизации ожидаемой полезности в случае экспоненциальных моделей Леви.

3.1. Описание модели. Предположим, что имеются два независимых $d$-мерных процесса Леви $X^{(1)}=\left(X_{t}^{(1)}\right)_{0 \leqslant t \leqslant T}$ и $X^{(2)}=\left(X_{t}^{(2)}\right)_{0 \leqslant t \leqslant T^{\prime}}$, выходящих из нуля, и имеющих, определяющие эти процессы триплеты характеристик $\left(b_{1}, c_{1}, K_{1}\right)$ и $\left(b_{2}, c_{2}, K_{2}\right)$ соответственно. Каждый такой процесс предполагается заданным на отвечающем ему каноническом вероятностном пространстве $\left(\Omega^{(1)}, \mathscr{F}^{(1)}, \mathbb{F}^{(1)}, P^{(1)}\right)$ и $\left(\Omega^{(2)}, \mathscr{F}^{(2)}, \mathbb{F}^{(2)}, P^{(2)}\right)$ соответственно, где $\mathbb{F}^{(1)}=\left(\mathscr{F}_{t}^{(1)}\right)_{0 \leqslant t \leqslant T}$ и $\mathbb{F}^{(2)}=\left(\mathscr{F}_{t}^{(2)}\right)_{0 \leqslant t \leqslant T^{\prime}}$ суть натуральные фильтрации процессов, удовлетворяющие стандартным условиям. Обозначим $X=\left(X_{t}\right)_{0 \leqslant t \leqslant T}$ линейную комбинацию процессов $X^{(1)}$ и $X^{(2)}$, более точно, для $t \in[0, T]$

$$
X_{t}=\rho_{1} X_{t}^{(1)}+\rho_{2} X_{t}^{(2)}
$$

где $\rho_{1}$ и $\rho_{2}$ - обратимые матрицы с действительными числовыми компонентами. Как уже было сказано, процесс $X$ будет рассматриваться на каноническом пространстве $(\Omega, \mathscr{F}, \mathbb{F}, P)$ с фильтрацией $\mathbb{F}=\left(\mathscr{F}_{t}\right)_{0 \leqslant t \leqslant T}$, удовлетворяющей стандартным условиям.

Рассмотрим также расширенное пространство $\left(\Omega \times \mathbf{R}^{d}, \mathscr{F} \otimes\right.$ $\left.\sigma\left(X_{T^{\prime}}^{(2)}\right), \mathbb{G}\right)$, отвечающее паре $\left(X, X_{T^{\prime}}^{(2)}\right)$ с расширенной фильтрацией $\mathbb{G}=$ $\left(\mathscr{G}_{t}\right)_{0 \leqslant t \leqslant T}$, где для $0 \leqslant t<T$

$$
\mathscr{G}_{t}=\bigcap_{s>t} \mathscr{F}_{s} \otimes \sigma\left(X_{T^{\prime}}^{(2)}\right) \quad \text { и } \quad \mathscr{G}_{T}=\mathscr{F}_{T} \otimes \sigma\left(X_{T^{\prime}}^{(2)}\right) .
$$

Заметим, что в пространстве $(\Omega, \mathscr{F}, P)$ процесс $X$, является, очевидно, процессом Леви. Если теперь мы введем на измеримом пространстве $\left(\Omega \times \mathbf{R}^{d}, \mathscr{F} \otimes \mathscr{B}\left(\mathbf{R}^{d}\right), \mathbb{G}\right)$ вероятностную меру $P \times \alpha$, где $\alpha$ есть закон распределения случайной величины $X_{T^{\prime}}^{(2)}$, то процесс $X$ останется процессом Леви с теми же характеристиками. Напомним, что как и ранее, совместное распределение пары $\left(X, X_{T^{\prime}}^{(2)}\right)$ обозначается $\mathbb{P}$, а условное распределения процесса $X$ при условии $X_{T^{\prime}}^{(2)}=v$ обозначается $P^{v}$. 
3.2. Предположения 1 и 2. В этой части нашей статьи мы покажем, что предположения 1 и 2, введенные в разд. 2, будут выполнены при следующих условиях на упомянутые выше процессы Леви.

У с л о в и е Н1. Процессы $X$ и $X^{(2)}$ являются интегрируемыми на $[0, T]$ и $\left[0, T^{\prime}\right]$ соответственно.

У с л о в и е Н2. Процесс $\left(X, X^{(2)}\right)$ имеет переходную плотность распределения по отношению к мере $\eta_{1} \times \eta_{2}$, являющейся прямым произведением $\sigma$-конечных мер $\eta_{1}$ и $\eta_{2}$ в пространстве $\left(\mathbf{R}^{d}, \mathscr{B}\left(\mathbf{R}^{d}\right)\right)$, a маргинальные переходные плотности распределения $f$ и $f^{(2)}$ процессов $X$ и $X^{(2)}$ по мерам $\eta_{1}$ и $\eta_{2}$ соответственно являются строго положительными.

3 а м е ч а н и е 2. Следует отметить, что в случае, когда $\eta_{1}$ и $\eta_{2}$ являются мерами Лебега, условие Н2 эквивалентно существованию маргинальных строго положительных переходных плотностей $f_{1}$ и $f_{2}$ распределений процессов $X^{(1)}$ и $X^{(2)}$. Этот факт вытекает из независимости процессов $X^{(1)}$ and $X^{(2)}$ и соответствующей формулы для вычисления плотности.

Предложение 4. При выполнении условий (H1) и (H2) имеют место предположения 1 и 2 разд. 2.1, а также существует функиия $F_{v}:\left[T^{\prime}-T, T^{\prime}\right] \times \mathbf{R}^{d} \rightarrow \mathbf{R}^{+}$, зависящая от параметра $v \in \mathbf{R}^{d}$, такая umo

$$
\frac{d \alpha^{t}}{d \alpha}(v)=\frac{F_{v}\left(T^{\prime}-t, X_{t}\right)}{F_{v}\left(T^{\prime}, 0\right)} .
$$

Более того,

$$
\frac{d \alpha^{t}}{d \alpha}=\mathscr{E}(M)_{t},
$$

где $M=\left(M_{t}\right)_{0 \leqslant t \leqslant T}$ есть не что иное как $(P, \mathbb{F})$ мартингал, такой, что

$$
M_{t}=\int_{0}^{t}{ }^{\top} \beta_{s}^{v, P} X_{s}^{c}+\int_{0}^{t} \int_{\mathbf{R}^{d}} l(x)\left(Y_{s}^{v, P}(x)-1\right) d K(x) d s,
$$

$a\left(\beta^{v, P}, Y^{v, P}\right)$ являются параметрами Гирсанова, отвечающими замене мерь $P$ на меру $P^{v}$, и $K$ - не что иное как мера Леви прочесса $X$.

Если $F_{v} \in C_{b}^{1,2}\left(\left[T^{\prime}-T, T^{\prime}\right] \times \mathbf{R}^{d}\right)$, а с- обратимая матрича, то упомянутые параметры Гирсанова могут быть вычислень по формулам:

$$
{ }^{\top} \beta_{s}^{v, P}=\left(\frac{\partial \ln F_{v}}{\partial x_{1}}\left(T^{\prime}-s, X_{s-}\right), \ldots, \frac{\partial \ln F_{v}}{\partial x_{d}}\left(T^{\prime}-s, X_{s-}\right)\right)
$$

$u \partial \Omega я x \in \mathbf{R}^{d} \backslash\{0\}$

$$
Y_{s}^{v, P}(x)=\frac{F_{v}\left(T-s, X_{s-}+x\right)}{F_{v}\left(T^{\prime}-s, X_{s-}\right)}
$$


Д о к а з а т е л ь с т в о. Условное распределение процесса $X$ при условии $X_{T^{\prime}}^{(2)}=v$ совпадает с распределением процесса $\rho_{1} X^{(1)}+\rho_{2} V^{(2)}$, где $V^{(2)}$ - мост Леви процесса Леви $X^{(2)}$ с начальной точкой $(0,0)$ и конечной точкой $\left(v, T^{\prime}\right)$. При выполнении условия (Н2) и согласно [12] закон распределения $\left(V_{t}^{(2)}\right)_{0 \leqslant t \leqslant T}$ абсолютно непрерывен по отношению к закону распределения $\left(X_{t}^{(2)}\right)_{0 \leqslant t \leqslant T}$ и

$$
\frac{d P_{V^{(2)}}}{d P_{X^{(2)}}}(T, v)=\frac{f^{(2)}\left(T^{\prime}-T, v-X_{T}^{(2)}\right)}{f^{(2)}\left(T^{\prime}, v\right)} .
$$

Поскольку процесс $X^{(1)}$ независим от $X^{(2)}$ и $V^{(2)}$, условное распределение процесса $X$ при условии $X^{(2)}$ и условное распределение $X$ при условии $V^{(2)}$ совпадают как отображения по вероятностной мере $P$. Обозначим это отображение $q(A, x)$, где $A \in \mathscr{F}_{T}, x \in \mathbf{R}^{d}$. Тогда

$$
\begin{aligned}
P(A) & =\int_{\mathbf{R}^{d}} P\left(\rho_{1} X^{(1)}+\rho_{2} X^{(2)} \in A \mid X^{(2)}=x\right) d P_{X^{(2)}}(x) \\
& =\int_{\mathbf{R}^{d}} q(A, x) d P_{X^{(2)}}(x)
\end{aligned}
$$

и

$$
\begin{aligned}
P^{v}(A) & =P\left(\rho_{1} X^{(1)}+\rho_{2} V^{(2)} \in A\right) \\
& =\int_{\mathbf{R}^{d}} P\left(\rho_{1} X^{(1)}+\rho_{2} V^{(2)} \in A \mid V^{(2)}=x\right) d P_{V^{(2)}}(x) \\
& =\int_{\mathbf{R}^{d}} q(A, x) \frac{d P_{V^{(2)}}}{d P_{X^{(2)}}}(T, v) d P_{X^{(2)}}(x) .
\end{aligned}
$$

Наконец, если $P(A)=0$, то $q(A, x)=0\left(P_{X^{(2)}}\right.$-п.н. $)$ и, значит, $P^{v}(A)=0$. А это означает, что предположение 1 выполняется.

Из предположения 1 и формулы Байеса в свою очередь следует, что

$$
\begin{aligned}
P\left(X_{T^{\prime}}^{(2)} \in d v \mid X_{t}=y\right) & =\frac{P\left(X_{T^{\prime}}^{(2)} \in d v, X_{t} \in d y\right)}{P\left(X_{t} \in d y\right)} \\
& =\frac{P\left(X_{t} \in d y \mid X_{T^{\prime}}^{(2)}=v\right) P\left(X_{T^{\prime}}^{(2)} \in d v\right)}{P\left(X_{t} \in d y\right)} .
\end{aligned}
$$

Отсюда следует, что и предположение 2 выполняется. Используя марковское свойство процесса $X^{(2)}$, получаем

$$
\begin{aligned}
\alpha^{t}(d v) & =P\left(X_{T^{\prime}}^{(2)} \in d v \mid \mathscr{F}_{t}\right)=P\left(X_{T^{\prime}}^{(2)} \in d v \mid X_{t}\right) \\
& =P\left(X_{T^{\prime}}^{(2)}-X_{t}^{(2)}+X_{t}^{(2)} \in d v \mid X_{t}\right) \\
& =P\left(\widetilde{X}_{T^{\prime}-t}^{(2)}+X_{t}^{(2)} \in d v\right),
\end{aligned}
$$


где $\widetilde{X}^{(2)}$ является процессом, независимым от $X^{(1)}$ и $X^{(2)}$, распределение которого совпадает с распределением процесса $X^{(2)}$. Проведенные рассуждения показывают, что $\alpha^{t}(d v)$ есть функция $T^{\prime}-t, X_{t}$ и параметра $v$. Обозначим эту функцию $F_{v}\left(T^{\prime}-t, X_{t}\right)$. Заметим, что $\alpha^{0}(d v)=\alpha(d v)=F_{v}\left(T^{\prime}, 0\right)$, поскольку $\mathscr{F}_{0}=\{\emptyset, \Omega\}$. Отсюда следует (3.2).

Теперь, используя формулу Ито, получаем

$$
\begin{aligned}
F_{v}\left(T^{\prime}-t, X_{t}\right)= & F_{v}\left(T^{\prime}, 0\right)-\int_{0}^{t} \frac{\partial F_{v}}{\partial s}\left(T^{\prime}-s, X_{s-}\right) d s \\
& +\sum_{i=1}^{d} \int_{0}^{t} \frac{\partial F_{v}}{\partial x_{i}}\left(T^{\prime}-s, X_{s-}\right) d X_{s}^{i} \\
& +\frac{1}{2} \sum_{i=1}^{d} \sum_{j=1}^{d} \int_{0}^{t} \frac{\partial^{2} F_{v}}{\partial x_{i} \partial x_{j}}\left(T^{\prime}-s, X_{s-}\right) d\left\langle X^{i, c}, X^{j, c}\right\rangle_{s} \\
& +\sum_{0<s \leqslant t} F_{v}\left(T^{\prime}-s, X_{s}\right)-F_{v}\left(T^{\prime}-s, X_{s-}\right) \\
& -\sum_{i=1}^{d} \frac{\partial F_{v}}{\partial x_{i}}\left(T^{\prime}-s, X_{s-}\right) \Delta X_{s}^{i} .
\end{aligned}
$$

При выполнении условия $P_{t}^{v} \ll P_{t}$ и $\alpha^{t} \ll \alpha$ для $\left.\left.t \in\right] 0, T\right]$ из леммы Жакода (см. [14]) вытекает, что $\left(d \alpha^{t} / d \alpha\right)_{0 \leqslant t \leqslant T}$ является $(P, \mathbb{F})$ мартингалом. Для упрощения обозначений положим $p_{t}^{v}=\left(d \alpha^{t} / d \alpha\right)(v)$ для $t \in[0, T]$. Теперь давайте разделим предыдущее выражение для $F_{v}\left(T^{\prime}-t, X_{t}\right)$ на $F_{v}\left(T^{\prime}, 0\right)$ и идентифицируем его непрерывную мартингальную часть. Таким образом, получаем, что

$$
p_{t}^{v, c}=\frac{1}{F_{v}\left(T^{\prime}, 0\right)} \sum_{i=1}^{d} \int_{0}^{t} \frac{\partial F_{v}}{d x_{i}}\left(T^{\prime}-s, X_{s-}\right) d X_{s}^{i, c},
$$

и, следовательно,

$$
\left\langle p^{v, c}, X^{j, c}\right\rangle_{t}=\frac{1}{F_{v}\left(T^{\prime}, 0\right)} \sum_{i=1}^{d} \int_{0}^{t} c_{i, j} \frac{\partial F_{v}}{d x_{i}}\left(T^{\prime}-s, X_{s-}\right) d s .
$$

Наконец, согласно теореме Гирсанова,

$$
\left\langle p^{v, c}, X^{j, c}\right\rangle_{t}=\sum_{i=1}^{d} \int_{0}^{t} c_{i, j}\left(\beta_{s}^{v, P}\right)^{i} p_{s-}^{v} d s .
$$

Поскольку матрица $c$ обратима, отсюда получаем формулу для $\beta_{t}^{v, P}$.

Теперь вычислим скачки $p^{v}$ :

$$
\Delta p_{t}^{v}=\frac{F_{v}\left(T^{\prime}-t, X_{t}\right)-F_{v}\left(T^{\prime}-t, X_{t-}\right)}{F_{v}\left(T^{\prime}, 0\right)}
$$


и

$$
\frac{\Delta p_{t}^{v}}{p_{t-}^{v}}=\frac{F_{v}\left(T^{\prime}-t, X_{t-}+\Delta X_{t}\right)}{F_{v}\left(T^{\prime}-t, X_{t-}\right)}-1 .
$$

Тогда согласно теореме 3.24 [16, с. 159]

$$
Y_{t}^{v, P}=\frac{\left.F_{v}\left(T^{\prime}-t, X_{t-}+x\right)\right)}{F_{v}\left(T^{\prime}-t, X_{t-}\right)}
$$

и, значит, наше утверждение доказано.

\section{3. Условные локально эквивалентные мартингальные} меры. Основная трудность, связанная с применением результатов разд. 2 состоит в проверке предположения 3. Чтобы осуществить такую проверку, сначала опишем множества условных локально эквивалентных маргинальных мер. Этот этап может быть осуществлен с помощью семимартингальной техники.

Напомним, что процесс $X$ определяется формулой (3.1). Как и ранее, обозначим $\left(\beta^{v, P}, Y^{v, P}\right)$ параметры Гирсанова, отвечающие замене меры $P$ на $P^{v} ; \mathscr{M}\left(P^{v}\right)$ - семейство локально эквивалентных по отношению к $P^{v}$ мартингальных мер $Q^{v}$. Обозначим $\left(\beta^{v}, Y^{v}\right)$ параметры Гирсанова, отвечающие замене меры $P^{v}$ на $Q^{v}$. Заметим, что $X$ является $\left(P^{v}, \mathbb{F}\right)$-семимартингалом, и, следовательно, также $\left(Q^{v}, \mathbb{F}\right)$-семимартингалом. В приведенном ниже предложении, дается триплет предсказуемых характеристик семимартингала $X$ по мере $Q^{v}$.

Предложение 5. Триплет $\left(B^{v}, C^{v}, \nu^{v}\right)$ предсказуемьх характеристик $\left(Q^{v}, \mathbb{F}\right)$-семимартингала $X$, определяется по следующим формулам:

$$
\begin{aligned}
B_{t}^{v}= & \left(\rho_{1} b_{1}+\rho_{2} b_{2}\right) t+\rho_{2} c_{2} \int_{0}^{t} \beta_{s}^{v, P} d s \\
& +\rho_{2} \int_{0}^{t} \int_{\mathbf{R}^{d}} \overrightarrow{l_{2}(x)}\left(Y_{s}^{v, P}\left(\rho_{2}^{-1} x\right)-1\right)\left(K_{2} \circ \rho_{2}^{-1}\right)(d x) d s \\
& +\int_{0}^{t} \int_{\mathbf{R}^{d}} \overrightarrow{(x)}\left(Y_{s}^{v}(x)-1\right) K_{s}^{v, P}(d x) d s \\
& +\left(\rho_{1} c_{1}^{\top} \rho_{1}+\rho_{2} c_{2}^{\top} \rho_{2}\right) \int_{0}^{t} \beta_{s}^{v} d s, \\
& C_{t}^{v}=\left(\rho_{1} c_{1}^{\top} \rho_{1}+\rho_{2} c_{2}^{\top} \rho_{2}\right) t, \\
& d \nu^{v}(x, s)=Y_{s}^{v} K_{s}^{v, P}(d x) d s,
\end{aligned}
$$

где $K_{s}^{v, P}(d x)=\left(K_{1} \circ \rho_{1}^{-1}\right)(d x)+Y_{s}^{v, P}\left(\rho_{2}^{-1} x\right)\left(K_{2} \circ \rho_{2}^{-1}\right)(d x)$.

Более того, эквивалентная по отношению $\kappa P_{T}^{v}$ маргинальная мера $Q_{T}^{v}$ определяется из следующего условия: для $s \in[0, T]$

$$
\begin{gathered}
\rho_{1} b_{1}+\rho_{2} b_{2}+\rho_{2} c_{2} \beta_{s}^{v, P}+\rho_{2} \int_{\mathbf{R}^{d}} \overrightarrow{l_{2}(x)}\left(Y_{s}^{v, P}\left(\rho_{2}^{-1} x\right)-1\right)\left(K_{2} \circ \rho_{2}^{-1}\right)(d x) \\
\quad+\int_{\mathbf{R}^{d}} \overrightarrow{(\vec{x})}\left(Y_{s}^{v}(x)-1\right) K_{s}^{v, P}(d x)+\left(\rho_{1} c_{1}^{\top} \rho_{1}+\rho_{2} c_{2}^{\top} \rho_{2}\right) \beta_{s}^{v}=0 .
\end{gathered}
$$


Д о к а з а т е л ь с т в о. Применим теорему Гирсанова для последовательной замены мер $P \rightarrow P^{v} \rightarrow Q^{v}$. Для этого напишем семимартингальное разложение процесса $X$ по исходной мере $P$ :

$X_{t}=B_{t}+X_{t}^{c}+\int_{0}^{t} \int_{\mathbf{R}^{d}} \overrightarrow{(x)}\left(\mu_{X}(d x, d s)-\nu_{X}(d x, d s)\right)+\sum_{s \leqslant t}\left(\Delta X_{s}-l\left(\Delta X_{s}\right)\right)$.

В этом разложении $B$ обозначает снос, $X^{c}$ является непрерывной маргинальной частью, а $\mu_{X}$ и $\nu_{X}$ обозначают меру скачков и отвечающий ей компенсатор, кроме того $l$ есть урезающая функция, $l(x)=x \mathbf{1}_{\{\|x\| \leqslant 1\}}, x \in$ $\mathbf{R}^{d}$. Следует также заметить, что интеграл на $\mathbf{R}^{d}$ в предшествующем выражении берется покомпонентно, а именно, для каждого $x \in \mathbf{R}^{d}$ и $l(x)={ }^{\top}\left(l^{1}(x), \ldots, l^{d}(x)\right)$ интеграл

$$
\int_{0}^{t} \int_{\mathbf{R}^{d}} \overrightarrow{l(x)}\left(\mu_{X}(d x, d s)-\nu_{X}(d x, d s)\right)
$$

есть вектор с компонентами

$$
\int_{0}^{t} \int_{\mathbf{R}^{d}} l^{i}(x)\left(\mu_{X}(d x, d s)-\nu_{X}(d x, d s)\right)
$$

где $1 \leqslant i \leqslant d$. Мы используем обозначения $\overrightarrow{l(x)}$, чтобы подчеркнуть упомянутую особенность интегрирования. $X^{(2)}$ :

Напишем также семимартингальные разложения процессов $X^{(1)}$ и

$$
\begin{aligned}
X_{t}^{(1)}= & b_{1} t+X_{t}^{(1), c}+\int_{0}^{t} \int_{\mathbf{R}^{d}} \overrightarrow{l_{1}(x)}\left(\mu_{X^{(1)}}(d x, d s)-K_{1}(d x) d s\right) \\
& +\sum_{s \leqslant t}\left(\Delta X_{s}^{(1)}-l_{1}\left(\Delta X_{s}^{(1)}\right)\right) \\
X_{t}^{(2)}= & b_{2} t+X_{t}^{(2), c}+\int_{0}^{t} \int_{\mathbf{R}^{d}} \overrightarrow{l_{2}(x)}\left(\mu_{X^{(2)}}(d x, d s)-K_{2}(d x) d s\right) \\
& +\sum_{s \leqslant t}\left(\Delta X_{s}^{(2)}-l_{2}\left(\Delta X_{s}^{(2)}\right)\right)
\end{aligned}
$$

с урезающими функциями $l_{1}(x)=x \mathbf{1}_{\left\{\left\|\rho_{1} x\right\| \leqslant 1\right\}}$ и $l_{2}(x)=x \mathbf{1}_{\left\{\left\|\rho_{2} x\right\| \leqslant 1\right\}}$ соответственно.

Теперь сравним линейную комбинацию семимартингальных разложений процессов $X^{(1)}$ и $X^{(2)}$, приведеных выше, с семимартингальным разложением самого процесса $X$. Мы можем легко идентифицировать снос процесса $X$, равный $\left(\rho_{1} b_{1}+\rho_{2} b_{2}\right) t, t \geqslant 0$, а также непрерывную мартингальную компоненту процесса $X$, которая равна $\rho_{1} X^{(1), c}+\rho_{2} X^{(2), c}$. Поскольку $X_{t}^{(1), c}$ и $X_{t}^{(2), c}$ являются независимыми процессами с квадратическими вариациями $c_{1} t, t \geqslant 0$, и $c_{2} t, t \geqslant 0$, то квадратическая вариация непрерывной мартингальной части процесса $X$ равна $\left(\rho_{1} c_{1}^{\top} \rho_{1}+\rho_{2} c_{2}^{\top} \rho_{2}\right) t, t \geqslant 0$. 
Для информации о скачкообразной компоненте процесса $X$, напишем меру скачков этого процесса:

$$
\mu_{X}(\omega, d t, d x)=\sum_{s} \mathbf{1}_{\left\{\Delta X_{s}(\omega) \neq 0\right\}} \delta_{\left\{\left(s, \Delta X_{s}(\omega)\right)\right\}}(d t, d x),
$$

где $\delta_{\left\{\left(s, \Delta X_{s}(\omega)\right)\right\}}$ - мера Дирака в точке $\left(s, \Delta X_{s}(\omega)\right)$. Кроме того,

$$
\Delta X=\rho_{1} \Delta X^{(1)}+\rho_{2} \Delta X^{(2)}, \quad l(\Delta X)=\rho_{1} l_{1}\left(\Delta X^{(1)}\right)+\rho_{2} l_{2}\left(\Delta X^{(2)}\right) .
$$

Известно, что скачки двух независимых процессов Леви не могут происходить одновременно. На самом деле, скачки процесса Леви являются тотально недостижимыми моментами остановки. Если предположить, что два независимых процесса Леви $X^{(1)}$ и $X^{(2)}$ имеют скачки в моменты $\tau_{1}$ и $\tau_{2}$ с $\tau_{1}=\tau_{2}$ (P-п.н.), тогда для всех $A \in \mathbf{R}^{d}$

$$
P\left(\left\{\tau_{1} \in A\right\} \cap\left\{\tau_{2} \in A\right\}\right)=P\left(\left\{\tau_{1} \in A\right\}\right)=P^{2}\left(\left\{\tau_{1} \in A\right\}\right) .
$$

Отсюда вытекает, что $P\left(\left\{\tau_{1} \in A\right\}\right)=0$ или 1 , и закон распределения $\tau_{1}$ может быть только мерой Дирака. Но тогда существует $t_{0} \in \mathbf{R}^{+}$ такое, что $P\left(\tau_{1}=t_{0}\right)=1$, а это противоречит тому, что $\tau_{1}$ тотально недостижим. Значит, $P$-п.н.

$$
\begin{aligned}
\left\{\Delta X_{s}(\omega) \neq 0\right\}= & \left\{\rho_{1} \Delta X_{s}^{(1)}(\omega) \neq 0\right\} \cup\left\{\rho_{2} \Delta X_{s}^{(2)}(\omega) \neq 0\right\} \\
= & \left\{\rho_{1} \Delta X_{s}^{(1)}(\omega) \neq 0\right\} \cap\left\{\Delta X_{s}^{(2)}(\omega)=0\right\} \\
& \cup\left\{\Delta X_{s}^{(1)}(\omega)=0\right\}\left\{\rho_{2} \Delta X_{s}^{(2)}(\omega) \neq 0\right\} .
\end{aligned}
$$

Как следствие, получаем, что

$$
\begin{aligned}
\mu_{X}(\omega, d t, d x)= & \sum_{s} \mathbf{1}_{\left\{\Delta X_{s}^{(1)}(\omega) \neq 0\right\}} \delta_{\left\{\left(s, \rho_{1} \Delta X_{s}^{(1)}(\omega)\right)\right\}}(d t, d x) \\
& +\sum_{s} \mathbf{1}_{\left\{\Delta X_{s}^{(2)}(\omega) \neq 0\right\}} \delta_{\left\{\left(s, \rho_{2} \Delta X_{s}^{(2)}(\omega)\right)\right\}}(d t, d x) \\
= & \mu_{\rho_{1} X^{(1)}}(\omega, d t, d x)+\mu_{\rho_{2} X^{(2)}}(\omega, d t, d x) .
\end{aligned}
$$

Далее, процессы $\rho_{1} X^{(1)}$ и $\rho_{2} X^{(2)}$ являются процессами Леви с отвечающими им мерами Леви $K_{1}\left(\rho_{1}^{-1} A\right)$ и $K_{2}\left(\rho_{2}^{-1} A\right)$ соответственно, где $A \in \mathscr{B}\left(\mathbf{R}^{d}\right)$. В связи с указанными фактами, триплет предсказуемых характеристик $(B, C, \nu)$ процесса $X$ определяется по следующим формулам:

$$
\begin{gathered}
B_{t}=\left(\rho_{1} b_{1}+\rho_{2} b_{2}\right) t, \\
C_{t}=\left(\rho_{1} c_{1}^{\top} \rho_{1}+\rho_{2} c_{2}^{\top} \rho_{2}\right) t, \\
d \nu(x, t)=\left(\left(K_{1} \circ \rho_{1}^{-1}\right)(d x)+\left(K_{2} \circ \rho_{2}^{-1}\right)(d x)\right) d t .
\end{gathered}
$$


Давайте теперь напишем формулы для семимартингальных характеристик $\left(B^{V}, C^{V}, \nu^{V}\right)$ моста Леви $V^{(2)}$ :

$$
\begin{gathered}
B_{t}^{V}=b_{2} t+c_{2} \int_{0}^{t} \beta_{s}^{v, P} d s+\int_{0}^{t} \int_{\mathbf{R}^{d}} \overrightarrow{l_{2}(x)}\left(Y_{s}^{v, P}(x)-1\right) K_{2}(d x) d s \\
C_{t}^{V}=c_{2} t \\
d \nu^{V}(x, t)=Y_{t}^{v, P}(x) K_{2}(d x) d t
\end{gathered}
$$

Наконец, чтобы написать семимартингальные характеристики линейной комбинации процессов $X^{(1)}$ и $V^{(2)}$, заметим, что процессы $X^{(1)}$ и $V^{(2)}$ являются независимыми по мере $P^{v}$. Поэтому, чтобы получить формулы для характеристик из предыдущих формул, достаточно добавить в формулу для сноса, дополнительный снос, возникающий от замены меры $P^{v}$ на меру $Q^{v}$, а также в формуле для компенсатора, умножить соответствующую меру Леви на $Y_{s}^{v}$. Таким образом, получаем указанные формулы для характеристик.

Процесс $X$ является, как известно, $\left(Q^{v}, \mathbb{F}\right)$-мартингалом тогда и только тогда, когда снос процесса по мере $Q^{v}$ равен нулю при всех значениях $t$. Таким образом, получаем указанную формулу (3.4).

3.4. Условные информационные процессы. Для упрощения обозначений положим

$$
b=\rho_{1} b_{1}+\rho_{2} b_{2}, \quad c=\rho_{1} c_{1}^{\top} \rho_{1}+\rho_{2} c_{2}^{\top} \rho_{2} .
$$

Напомним, что $(b, c, K)$ являются параметрами процесса Леви $X$ по так называемой наблюдаемой мере $P$.

Теорема 3. Предположим, что $u(x)=\ln x$ и что условия $(\mathrm{H} 1)$ u (Н2) выполнень. Если существует предсказуемый прочесс $\lambda^{v}=$ $\left(\lambda_{s}^{v}\right)_{0 \leqslant s \leqslant T}$ со значениями в $\mathbf{R}^{d}$ такой, что для всех $s \in[0, T]$

$$
\begin{gathered}
b+c \lambda_{s}^{v}+\rho_{2} c_{2} \beta_{s}^{v, P}+\rho_{2} \int_{\mathbf{R}^{d}} \overrightarrow{l_{2}(x)}\left[Y_{s}^{v, P}\left(\rho_{2}^{-1} x\right)-1\right]\left(K_{2} \circ \rho_{2}^{-1}\right)(d x) \\
+\int_{\mathbf{R}^{d}} \overrightarrow{(x)} \frac{{ }^{\top} \lambda_{s}^{v} l(x)}{1-{ }^{\top} \lambda_{s}^{v} l(x)} K_{s}^{v, P}(d x)=0,
\end{gathered}
$$

и такой, что $1-{ }^{\top} \lambda_{s}^{v} l(x)>0$ (K ${ }^{v, P}$-a.s. $)$, то параметры Гирсанова эквивалентной, мартингальной, минимизирующей $f$-дивергенцию меры $Q_{T}^{v, *}$ определяются из следуюших соотношений:

$$
\beta_{s}^{v, *}=\lambda_{s}^{v}, \quad Y_{s}^{v, *}(x)=\frac{1}{1-{ }^{\top} \lambda_{s}^{v} l(x)} .
$$

Соответствующий информационный процесс $\mathscr{I}^{*}(v)$ определяется по формуле (2.7), а соответствуюшая ему энтропия, по формуле (2.8). Если отвечающая информачионному прочессу энтропия конечна, то полученная мера будет эквивалентной, мартингальной и минимальной. 
Д о к а з а т е л ь с т в о. Чтобы определить параметры Гирсанова эквивалентной, мартингальной и минимальной по отношению к соответствующей $f$-дивергенции меры, будем минимизировать энтропию меры $P_{T}^{v}$ по отношению к мере $Q_{T}^{v}$ :

$$
\mathbf{I}\left(P_{T}^{v} \mid Q_{T}^{v}\right)=\mathbf{E}_{P_{T}^{v}}\left(\mathscr{I}_{T}(v)\right)
$$

где

$$
\mathscr{I}_{T}(v)=\frac{1}{2} \int_{0}^{T}{ }^{\top} \beta_{s}^{v} c \beta_{s}^{v} d s-\int_{0}^{T} \int_{\mathbf{R}^{d}}\left(\ln Y_{s}^{v}(x)-Y_{s}^{v}+1\right) K_{s}^{v, P}(d x) d s,
$$

с ограничением, таким, что для $s \in[0, T]$

$$
R\left(\beta_{s}^{v}, Y_{s}^{v}\right)=0 .
$$

Входящая в ограничение функция $R\left(\beta_{s}^{v}, Y_{s}^{v}\right)$ определяется по формуле

$$
\begin{aligned}
R\left(\beta_{s}^{v}, Y_{s}^{v}\right)= & b+\rho_{2} c_{2} \beta_{s}^{v, P}+\int_{\mathbf{R}^{d}} l \overrightarrow{(x)}\left[Y_{s}^{v, P}\left(\rho_{2}^{-1} x\right)-1\right]\left(K_{2} \circ \rho_{2}^{-1}\right)(d x) \\
& +c \beta_{s}^{v}+\int_{\mathbf{R}^{d}} l \overrightarrow{(x)}\left(Y_{s}^{v}(x)-1\right) K_{s}^{v, P}(d x) .
\end{aligned}
$$

Согласно традиционным подходам к задаче минимизации, введем функцию

$G\left(\beta_{s}^{v}, Y_{s}^{v}\right)=\frac{1}{2}{ }^{\top} \beta_{s}^{v} c \beta_{s}^{v}-\int_{\mathbf{R}^{d}}\left(\ln \left(Y_{s}^{v}(x)\right)-Y_{s}^{v}+1\right) K_{s}^{v, P}(d x)-{ }^{\top} \lambda_{s}^{v} R\left(\beta_{s}^{v}, Y_{s}^{v}\right)$,

где $\lambda_{s}^{v}$ - множитель Лагранжа. Указанная функция является выпуклой непрерывно дифференцируемой функцией от $\left(\beta_{s}^{v}, Y_{s}^{v}\right)$, и, значит, экстремальными точками могут быть только стационарные точки этой функции, определяемые из следующей системы уравнений:

$$
\left\{\begin{array}{l}
\top\left(\frac{\partial G}{\partial \beta_{1}}\left(\beta_{s}^{v}, Y_{s}^{v}\right), \ldots, \frac{\partial G}{\partial \beta_{d}}\left(\beta_{s}^{v}, Y_{s}^{v}\right)\right)=c\left(\beta_{s}^{v}-\lambda_{s}^{v}\right)=0, \\
\frac{\partial G}{\partial Y}\left(\beta_{s}^{v}, Y_{s}^{v}\right)=\int_{\mathbf{R}^{d}}\left(1-\frac{1}{Y_{s}^{v}(x)}-{ }^{\top} \lambda_{s}^{v} l(x)\right) K_{s}^{v, P}(d x)=0 .
\end{array}\right.
$$

Из первого уравнения системы ясно, что $\beta_{s}^{v}=\lambda_{s}^{v}$. Решение второго уравнения неединственно. Возьмем одно из его решений, а именно

$$
Y_{s}^{v}(x)=\frac{1}{1-{ }^{\top} \lambda_{s}^{v} l(x)} .
$$

В силу выпуклости функции $G$, значение этой функции во всех стационарных точках одинаково. Давайте теперь подставим найденные параметры $\beta_{s}^{v}$ и $Y_{s}^{v}$ в мартингальное условие (3.5), найдем $\lambda_{s}^{v}$, и, следовательно, $\beta^{v, *}$ и $Y^{v, *}$. 
Поскольку функция $G$ выпуклая, получаем

$$
\begin{aligned}
& G\left(\beta_{s}^{v}, Y_{s}^{v}\right)-G\left(\beta_{s}^{v, *}, Y_{s}^{v, *}\right) \\
& \geqslant^{\top}\left(\frac{\partial G}{\partial \beta_{1}}\left(\beta_{s}^{v, *}, Y_{s}^{v, *}\right), \ldots, \frac{\partial G}{\partial \beta_{d}}\left(\beta_{s}^{v, *}, Y_{s}^{v, *}\right)\right)\left(\beta_{s}^{v}-\beta_{s}^{v, *}\right) \\
& \quad+\frac{\partial G}{\partial Y}\left(\beta_{s}^{v, *}, Y_{s}^{v, *}\right)\left(Y_{s}^{v}-Y_{s}^{v}\right)=0 .
\end{aligned}
$$

Теперь проинтегрируем приведенное выше неравенство по $s$ и возьмем математическое ожидание по мере $P_{T}^{v}$. В результате получим, что соответствующая мера является минимальной, т.е.

$$
\mathbf{I}\left(P_{T}^{v} \mid Q_{T}^{v}\right) \geqslant \mathbf{I}\left(P_{T}^{v} \mid Q_{T}^{v, *}\right)
$$

Теорема 4. Предположим, что $u(x)=x \ln (x)+x-1$ и что условия (H1) и (Н2) вьполняются. Тогда в случае, когда существует предсказуемьй процесс $\lambda^{v}=\left(\lambda_{s}^{v}\right)_{0 \leqslant s \leqslant T}$ со значениями в $\mathbf{R}^{d}$ такой, что для всех $s \in[0, T]$

$$
\begin{gathered}
b+c \lambda_{s}^{v}+\rho_{2} c_{2} \beta_{s}^{v, P}+\rho_{2} \int_{\mathbf{R}^{d}} \overrightarrow{l_{2}(x)}\left[Y_{s}^{v, P}\left(\rho_{2}^{-1} x\right)-1\right]\left(K_{2} \circ \rho_{2}^{-1}\right)(d x) \\
+\int_{\mathbf{R}^{d}} \overrightarrow{(x)}\left[\exp \left({ }^{\top} \lambda_{s}^{v} l(x)\right)-1\right] K_{s}^{v, P}(d x)=0,
\end{gathered}
$$

параметры Гирсанова эквивалентной, мартингальной, минимизирующей $f$-дивергенцию мерь $Q_{T}^{v, *}$ вычисляются по формуле:

$$
\beta_{s}^{v, *}=\lambda_{s}^{v}, \quad Y_{s}^{v, *}(x)=\exp \left({ }^{\top} \lambda_{s}^{v} l(x)\right)
$$

Более того, соответствующий информачионный проџесс $I^{*}(v)$ задается формулой (2.9), а отвечающая ему информачия КульбакаЛейблера определяется из (2.10). Если полученная информаиия Кульбака-Лейблера конечна, то найденная мера будет эквивалентной, мартингальной, минимальной мерой.

Д о к а з а т е л с с в о. Чтобы найти параметры Гирсанова минимальной, мартингальной меры, минимизируется информация Кульбака-Лейблера меры $Q_{T}^{v}$ по отношению к мере $P_{T}^{v}$, а именно

$$
\mathbf{I}\left(Q_{T}^{v} \mid P_{T}^{v}\right)=\mathbf{E}_{Q_{T}^{v}}\left(I_{T}(v)\right)
$$

где

$I_{T}(v)=\frac{1}{2} \int_{0}^{T}{ }^{\top} \beta_{s}^{v} c \beta_{s}^{v} d s+\int_{0}^{T} \int_{\mathbf{R}^{d}}\left(Y_{s}^{v}(x) \ln \left(Y_{s}^{v}(x)\right)-Y_{s}^{v}+1\right) K_{s}^{v, P}(d x) d s$ 
с ограничением (3.6). Для этого вводится функция

$$
\begin{aligned}
G\left(\beta_{s}^{v}, Y_{s}^{v}\right)= & \frac{1}{2}{ }^{\top} \beta_{s}^{v} c \beta_{s}^{v} \\
& +\int_{\mathbf{R}^{d}}\left(Y_{s}^{v}(x) \ln \left(Y_{s}^{v}(x)\right)-Y_{s}^{v}+1\right) K_{s}^{v, P}(d x)-{ }^{\top} \lambda_{s}^{v} R\left(\beta_{s}^{v}, Y_{s}^{v}\right)
\end{aligned}
$$

с множителем Лагранжа $\lambda_{s}^{v}$. Поскольку эта функция является выпуклой непрерывно-дифференцируемой функцией, экстремальные точки являются стационарными точками этой функции. В свою очередь, стационарные точки определяются из следующей системы уравнений:

$$
\left\{\begin{array}{l}
\top\left(\frac{\partial G}{\partial \beta_{1}}\left(\beta_{s}^{v}, Y_{s}^{v}\right), \ldots, \frac{\partial G}{\partial \beta_{d}}\left(\beta_{s}^{v}, Y_{s}^{v}\right)\right)=c\left(\beta_{s}^{v}-\lambda_{s}^{v}\right)=0, \\
\frac{\partial G}{\partial Y}\left(\beta_{s}^{v}, Y_{s}^{v}\right)=\int_{\mathbf{R}^{d}}\left(\ln \left(Y_{s}^{v}(x)\right)-{ }^{\top} \lambda_{s}^{v} l(x)\right) K_{s}^{v, P}(d x)=0 .
\end{array}\right.
$$

Из первого уравнения находим, что $\beta_{s}^{v}=\lambda_{s}^{v}$. Как уже отмечалось, решение второго уравнения не единственно, а одно из решений будет равно

$$
Y_{s}^{v}(x)=\exp \left({ }^{\top} \lambda_{s}^{v} l(x)\right)
$$

При таком выборе стационарной точки, процесс $\lambda^{v}$, находится из условия (3.7).

Из выпуклости функции $G$ получаем, что

$$
\begin{aligned}
& G\left(\beta_{s}^{v}, Y_{s}^{v}\right)-G\left(\beta_{s}^{v, *}, Y_{s}^{v, *}\right) \\
& \geqslant^{\top}\left(\frac{\partial G}{\partial \beta_{1}}\left(\beta_{s}^{v, *}, Y_{s}^{v, *}\right), \ldots, \frac{\partial G}{\partial \beta_{d}}\left(\beta_{s}^{v, *}, Y_{s}^{v, *}\right)\right)\left(\beta_{s}^{v}-\beta_{s}^{v, *}\right) \\
& \quad+\frac{\partial G}{\partial Y}\left(\beta_{s}^{v, *}, Y_{s}^{v, *}\right)\left(Y_{s}^{v}-Y_{s}^{v}\right)=0 .
\end{aligned}
$$

Далее, чтобы показать, что найденная мера минимальная, предыдущее неравенство интегрируется по $s$, затем берется математическое ожидание по мере $Q_{T}^{v}$. В результате, получаем, что

$$
\mathbf{I}\left(Q_{T}^{v} \mid P_{T}^{v}\right) \geqslant \mathbf{I}\left(Q_{T}^{v, *} \mid P_{T}^{v}\right) .
$$

Теорема 5. Предположим, что $u(x)=x^{p}, p<1$, и что выполнень условия (H1) и (Н2). В случае, когда существует предсказуемьии прочесс $\lambda^{v}=\left(\lambda_{s}^{v}\right)_{0 \leqslant s \leqslant T}$ со значениями в $\mathbf{R}^{d}$ такой, что для всех $s \in[0, T]$ $u q=p /(p-1)$

$$
\begin{gathered}
b+\frac{c \lambda_{s}^{v}}{q(1-q)}+\rho_{2} c_{2} \beta_{s}^{v, P}+\rho_{2} \int_{\mathbf{R}^{d}} \overrightarrow{l_{2}(x)}\left[Y_{s}^{v, P}\left(\rho_{2}^{-1} x\right)-1\right]\left(K_{2} \circ \rho_{2}^{-1}\right)(d x) \\
+\int_{\mathbf{R}^{d}} \overrightarrow{(x)}\left[\left(1-\frac{\lambda_{s}^{v} l(x)}{q}\right)^{1 /(q-1)}-1\right] K_{s}^{v, P}(d x)=0
\end{gathered}
$$


u, такой, что $1-{ }^{\top} \lambda_{s}^{v} l(x) / q>0\left(K^{v, P}\right.$-а.s. $)$, параметры Гирсанова соответствующей эквивалентной, мартингальной, минимизирующей $f$ дивергениию мерь $Q_{T}^{v, *}$ определяются по следующим формулам:

$$
\beta_{s}^{v, *}=\frac{1}{q(1-q)} \lambda_{s}^{v}, \quad Y_{s}^{v, *}(x)=\left(1-\frac{{ }^{\top} \lambda_{s}^{v} l(x)}{q}\right)^{1 /(q-1)} .
$$

Более того, соответствующий процесс Хеллингера определяется из (2.11), а отвечающий процессу интеграл Хеллингера вычисляется по формуле (2.13). Если полученный интеграл Хеллингера конечен, то найденная мера будет эквивалентной, мартингальной, минимальной мерой.

Д о к а з а т е л ь с т в о. Чтобы найти параметры Гирсанова минимальной мартингальной меры, минимизируем интеграл Хеллингера мер $Q_{T}^{v}$ и $P_{T}^{v}$

$$
\mathbf{H}_{T}^{(q)}(v)=\mathbf{E}_{R_{T}^{v}} \exp \left(-h_{T}^{(q)}(v)\right)
$$

где

$$
\begin{aligned}
h_{T}^{(q)}(v)= & \frac{q(1-q)}{2} \int_{0}^{T}{ }^{\top} \beta_{s}^{v} c \beta_{s}^{v} d s \\
& -\int_{0}^{T} \int_{\mathbf{R}^{d}}\left(\left(Y_{s}^{v}(x)\right)^{q}-q\left(Y_{s}^{v}-1\right)-1\right) K_{s}^{v, P}(d x) d s
\end{aligned}
$$

с ограничением (3.6). Для этого введем функцию

$$
\begin{aligned}
G\left(\beta_{s}^{v}, Y_{s}^{v}\right)= & \frac{q(1-q)}{2}{ }^{\top} \beta_{s}^{v} c \beta_{s}^{v} d s \\
& -\int_{\mathbf{R}^{d}}\left(\left(Y_{s}^{v}(x)\right)^{q}-q\left(Y_{s}^{v}-1\right)-1\right) K_{s}^{v, P}(d x)-{ }^{\top} \lambda_{s}^{v} R\left(\beta_{s}^{v}, Y_{s}^{v}\right),
\end{aligned}
$$

где $\lambda_{s}^{v}$ - множитель Лагранжа. Заметим, что функция $G$ - выпуклая и непрерывно-дифференцируемая. Для нахождения минимума функции, найдем ее стационарные точки, являющиеся решением следующей системы уравнений:

$$
\left\{\begin{array}{l}
\top\left(\frac{\partial G}{\partial \beta_{1}}\left(\beta_{s}^{v}, Y_{s}^{v}\right), \ldots, \frac{\partial G}{\partial \beta_{d}}\left(\beta_{s}^{v}, Y_{s}^{v}\right)\right)=c\left(q(1-q) \beta_{s}^{v}-\lambda_{s}^{v}\right)=0, \\
\frac{\partial G}{\partial Y}\left(\beta_{s}^{v}, Y_{s}^{v}\right)=-\int_{\mathbf{R}^{d}}\left[q\left(Y_{s}^{v}(x)\right)^{q-1}-q+{ }^{\top} \lambda_{s}^{v} l(x)\right] K_{s}^{v, P}(d x)=0 .
\end{array}\right.
$$

Из первого уравнения находим, что $\beta_{s}^{v}=\lambda_{s}^{v} /(q(1-q))$. Одним из решений второго уравнения является

$$
Y_{s}^{v}(x)=\left(1-\frac{{ }^{\top} \lambda_{s}^{v} l(x)}{q}\right)^{1 /(q-1)}
$$


Подставим теперь найденные значения в уравнение (3.7), чтобы найти $\lambda_{s}^{v}$, а следовательно, и $\beta_{s}^{v, *}$ и $Y_{s}^{v, *}$.

Поскольку функция $G$ выпуклая,

$$
\begin{aligned}
& G\left(\beta_{s}^{v}, Y_{s}^{v}\right)-G\left(\beta_{s}^{v, *}, Y_{s}^{v, *}\right) \\
& \geqslant \geqslant^{\top}\left(\frac{\partial G}{\partial \beta_{1}}\left(\beta_{s}^{v, *}, Y_{s}^{v, *}\right), \ldots, \frac{\partial G}{\partial \beta_{d}}\left(\beta_{s}^{v, *}, Y_{s}^{v, *}\right)\right)\left(\beta_{s}^{v}-\beta_{s}^{v, *}\right) \\
& \quad+\frac{\partial G}{\partial Y}\left(\beta_{s}^{v, *}, Y_{s}^{v, *}\right)\left(Y_{s}^{v}-Y_{s}^{v}\right)=0 .
\end{aligned}
$$

Теперь нам остается лишь проинтегрировать полученное выше неравенство по $s$, учесть,что экспоненциальная функция также выпукла, и вычислить математическое ожидание по мере $R_{T}^{v}$. В итоге получаем, что $\mathbf{H}_{T}^{(q)} \geqslant \mathbf{H}_{T}^{(q), *}$, а это означает, что мера $Q_{T}^{v, *}$ является минимальной.

\section{4. Модель Блэка и Шоулса с коррелированными броунов-} скими движениями. Обозначим $\left(W^{(1)}, W^{(2)}\right)$ два независимых стандартных броуновских движения. Пусть также $\mu_{1}, \mu_{2} \in \mathbf{R}$ и $\sigma_{1}>0$, $\sigma_{2}>0$. Положим

$$
X_{t}^{(1)}=\mu_{1}+\sigma_{1} W_{t}^{(1)}, \quad X_{t}^{(2)}=\mu_{2}+\sigma_{2} W_{t}^{(2)},
$$

и, кроме того, выбрав $\rho,|\rho| \leqslant 1$, рассмотрим процесс

$$
X_{t}=\sqrt{1-\rho^{2}} X_{t}^{(1)}+\rho X_{t}^{(2)} .
$$

Полученный процесс $X$ будет броуновским движением с коэффициентом сноса

$$
\mu=\sqrt{1-\rho^{2}} \mu_{1}+\rho \mu_{2},
$$

коэффициентом диффузии

$$
\sigma^{2}=\left(1-\rho^{2}\right) \sigma_{1}^{2}+\rho^{2} \sigma_{2}^{2}
$$

и коэффициентом корреляции между $X_{t}$ и $X_{t}^{(2)}$ равным $\rho$. Заметим, что для вычисления условного математического ожидания, можно взять $W_{T^{\prime}}^{(2)}$ вместо $X_{T^{\prime}}^{(2)}$, поскольку эти случайные величины генерируют одну и ту же сигма-алгебру. Но такая замена должна сопровождаться также заменой функции $g(v)$ на $\widetilde{g}(v)=\exp \left\{\left(\mu_{2} T^{\prime}+\sigma_{2} v\right\}\right.$ в формуле, определяющей максимум ожидаемой полезности. В нашем примере закон распределения $\alpha$, очевидно, не что иное, как $\mathscr{N}\left(0, T^{\prime}\right)$.

Покажем, что условия (H1) и (Н2) выполнены. На самом деле, процессы $X^{(1)}$ и $X^{(2)}$ интегрируемы, и оба имеют строго положительную плотность распределения по отношению к мере Лебега. В частности, 
как известно, $W_{t}^{(2)}$ имеет строго положительную плотность распределения по мере Лебега для $t>0$

$$
f(t, x)=\frac{1}{\sqrt{2 \pi t}} \exp \left\{-\frac{x^{2}}{2 t}\right\},
$$

принадлежащую к классу $C_{b}^{1,2}([\epsilon, \infty[)$ для любого $\epsilon>0$. Кроме того, используя теорему о нормальной корреляции, получаем, что

$$
p_{T}^{v}(X)=\frac{d P_{T}^{v}}{d P_{T}}(X)=\left(\frac{T^{\prime}}{T^{\prime}-\rho^{2} T}\right)^{1 / 2} \exp \left\{-\frac{1}{2}\left[\frac{\left(v-\rho X_{T}\right)^{2}}{T^{\prime}-\rho^{2} T}-\frac{v^{2}}{T^{\prime}}\right]\right\} .
$$

Запишем указанную плотность как стохастическую экспоненту

$$
p_{T}^{v}(X)=\exp \left\{\int_{0}^{T} \beta_{s}^{v, P} d X_{s}^{c}-\frac{1}{2} \int_{0}^{T}\left(\beta_{s}^{v, P}\right)^{2} d s\right\}
$$

где $X$ является каноническим процессом, чтобы получить, что

$$
\beta_{t}^{v, P}=\rho \frac{v-\rho X_{t}^{c}}{T^{\prime}-\rho^{2} t} .
$$

Теперь нам остается вычислить информационные количества, рассмотренные в разд. 2. Результаты вычислений приводятся ниже.

Предложение 6 (см. [10]). Информационнье количества, такие как энтропия, информация Кульбака-Лейблера, интеграль Хеллингера, въчисляются по формулам

$$
\begin{aligned}
\mathbf{I}\left(P^{v} \mid Q^{v, *}\right)= & \frac{1}{2} \ln \left(\frac{T^{\prime}}{T^{\prime}-\rho^{2} T}\right)+\frac{T}{2}\left(\frac{\mu}{\sigma}+\frac{\rho v}{T^{\prime}}\right)^{2}-\frac{\rho^{2} T}{2 T^{\prime}}, \\
\mathbf{I}\left(Q^{v, *} \mid P^{v}\right)= & -\frac{1}{2} \ln \left(\frac{T^{\prime}}{T^{\prime}-\rho^{2} T}\right)+\frac{T T^{\prime}}{2\left(T^{\prime}-\rho^{2} T\right)}\left(\frac{\mu}{\sigma}+\frac{\rho v}{T^{\prime}}\right)^{2}+\frac{\rho^{2} T}{2\left(T^{\prime}-\rho^{2} T\right)}, \\
\mathbf{H}_{T}^{(q)}(v)= & \left(\frac{T^{\prime}}{T^{\prime}-q \rho^{2} T}\right)^{1 / 2}\left(\frac{T^{\prime}-\rho^{2} T}{T^{\prime}}\right)^{q / 2} \\
& \times \exp \left\{-\frac{q(1-q) T}{2\left(T^{\prime}-q \rho^{2} T\right)}\left(\frac{\mu}{\sigma}+\frac{\rho v}{T^{\prime}}\right)^{2}\right\} .
\end{aligned}
$$

Далее, чтобы получить максимальную полезность, достаточно использовать теорему 2 из разд. 2 с законом распределения $\alpha$, равным $\mathscr{N}\left(0, T^{\prime}\right)$.

5. Пример модели со скачками. Пусть $\left(W^{(1)}, W^{(2)}\right)$ обозначают пару стандартных броуновских движений с коэффициентом корреляции $\rho,|\rho| \leqslant 1$. Пусть также $N$ является однородным пуассоновским процессом с интенсивностью $\lambda>0$, независимым от $\left(W^{(1)}, W^{(2)}\right)$. Положим

$$
\begin{gathered}
X_{t}=\mu_{1} t+\sigma_{1} W_{t}^{(1)}+N_{t}, \quad t \in[0, T], \\
X_{t}^{(2)}=\mu_{1} t+\sigma_{1} W_{t}^{(2)}, \quad t \in\left[0, T^{\prime}\right],
\end{gathered}
$$


где $T^{\prime}>T$. В этом примере опцион будет участвовать в полезности как $g\left(X_{T^{\prime}}^{(2)}\right)$, где $g$ является измеримой неотрицательный функцией на $\mathbf{R}$.

По тем же соображениям, что и в разд. 4 , возьмем $W_{T^{\prime}}^{(2)}$ вместо $X_{T^{\prime}}^{(2)}$ с соответствующей заменой $g(v)$ на $\widetilde{g}(v)=\exp \left\{\left(\mu_{1} T^{\prime}+\sigma_{1} v\right\}\right.$. Так же как и в предыдущем примере, нетрудно проверить, что условия (Н1) и (Н2) выполнены. Более того,

$$
p_{T}^{v}(X)=\frac{d P_{T}^{v}}{d P_{T}}(X)=\left(\frac{T^{\prime}}{T^{\prime}-\rho^{2} T}\right)^{1 / 2} \exp \left\{-\frac{1}{2}\left[\frac{\left(\sigma_{1} v-\rho X_{T}^{c}\right)^{2}}{\sigma_{1}^{2}\left(T^{\prime}-\rho^{2} T\right)}-\frac{v^{2}}{T^{\prime}}\right]\right\}
$$

где $X$ - канонический процесс, отвечающий $X^{(1)}$, а $X^{c}-$ его непрерывная мартингальная часть. Запишем приведенное выше выражение в виде стохастический экспоненты, чтобы получить, что $P$-п.н. для всех $t \in[0, T]$

$$
\beta_{t}^{v, P}=\frac{\rho\left(v \sigma_{1}-\rho X_{t}^{c}\right)}{\sigma_{1}^{2}\left(T^{\prime}-\rho^{2} t\right)} .
$$

Заметим, что в нашем случае $Y^{v, P}=1$, так как процессы $N$ и $W^{(2)}$ независимы.

В следующей лемме приводятся выражения для параметров Гирсанова $\left(\beta^{v, *}, Y^{v, *}\right)$, отвечающих замене меры $P^{v}$ на $Q^{v, *}$.

Лемма 1. Параметры Гирсанова $\left(\beta^{v, *}, Y^{v, *}\right)$ эквивалентной, мартингальной, минимальной по отношению $\kappa f$-дивергенции мерь $Q_{T}^{v, *}$ определяются из следуюших соотношений:

1) для логарифмической полезности и $f(x)=-\ln x$

$$
\frac{\lambda}{\sigma_{1}^{2}}\left(Y_{t}^{v, *}-1\right)+\frac{\mu_{1}}{\sigma_{1}^{2}}+\beta_{t}^{v, P}-\frac{1}{Y_{t}^{v, *}}+1=0, \quad \beta_{t}^{v, *}=1-\frac{1}{Y_{t}^{v, *}},
$$

2) для экспоненциальный полезности и $f(x)=x \ln x-x+1$

$$
\frac{\lambda}{\sigma_{1}^{2}}\left(Y_{t}^{v, *}-1\right)+\frac{\mu_{1}}{\sigma_{1}^{2}}+\beta_{t}^{v, P}+\ln Y_{t}^{v, *}=0, \quad \beta_{t}^{v, *}=\ln Y_{t}^{v, *},
$$

3) для степенной полезности и $f(x)=-x^{q} / q$

$$
\begin{gathered}
\frac{\lambda}{\sigma_{1}^{2}}\left(Y_{t}^{v, *}-1\right)+\frac{\mu_{1}}{\sigma_{1}^{2}}+\beta_{t}^{v, P}+\frac{1}{1-q}\left[1-\left(Y_{t}^{v, *}\right)^{q-1}\right]=0, \\
\beta_{t}^{v, *}=\frac{1}{1-q}\left[1-\left(Y_{t}^{v, *}\right)^{q-1}\right] .
\end{gathered}
$$

Д о к а з а т е л ь с т в о. Указанные результаты вытекают из теорем 3,4 и 5. Для этого, выражаем $\lambda_{s}^{v}$ через $Y_{s}^{v, *}$, и затем заменяем $b$ на $\mu_{1}, c$ и $c_{2}$ на $\sigma_{1}^{2}$, и подставляем компенсатор меры скачков процесса $N$ равный $\lambda \delta_{\{1\}}$, где $\delta_{\{1\}}$ есть дельта-функции Дирака в точке 1 . Лемма доказана. 
Введем новую выпуклую функцию $\widehat{f}$ такую, что $\widehat{f}(x)=f(x)+x^{2} / 2$. Обозначим $\widehat{u}$ дуальную к $\widehat{f}$ функцию, в смысле преобразования ФенхеляЛежандра. Наконец, обозначим $\widehat{I}=\left(-\widehat{f}^{\prime}\right)^{-1}$ производную функции $\widehat{u}$.

Предложение 7. Получаем следующие выражения для $Y^{v, *}$ :

1) в случае логарифмической полезности

$$
Y_{t}^{v, *}=\frac{\sigma_{1}}{\sqrt{\lambda}} \widehat{I}\left(\frac{\sigma_{1}}{\sqrt{\lambda}}\left(\beta_{t}^{v, P}+\frac{\mu_{1}}{\sigma_{1}^{2}}+1-\frac{\lambda}{\sigma_{1}^{2}}\right)\right),
$$

2) в случае экспоненциальный полезности

$$
Y_{t}^{v, *}=\frac{\sigma_{1}^{2}}{\lambda} \widehat{I}\left(\beta_{t}^{v, P}+\frac{\mu_{1}}{\sigma_{1}^{2}}+\ln \frac{\sigma_{1}^{2}}{\lambda}-\frac{\lambda}{\sigma_{1}^{2}}\right),
$$

3) в случае степенной полезности

$$
\begin{aligned}
Y_{t}^{v, *}= & \left(\frac{\sigma_{1}^{2}}{(1-q) \lambda}\right)^{1 /(2-q)} \\
& \times \widehat{I}\left(\left(\frac{\sigma_{1}^{2}}{(1-q) \lambda}\right)^{(1-q) /(2-q)}\left[(1-q)\left(\beta_{t}^{v, P}+\frac{\mu_{1}}{\sigma_{1}^{2}}-\frac{\lambda}{\sigma_{1}^{2}}\right)+1\right]\right) .
\end{aligned}
$$

Д о к а з а т е ль с т в о. Для доказательства этих формул, воспользуемся предыдущей леммой. Чтобы найти $Y=Y^{v, *}$, введем новую функцию $U$ такую, что $Y=c U$, затем выберем $c$, чтобы выразить левую часть уравнений через функцию $\widehat{I}$.

Предложение 8. Для введенных ранее информационньх количеств получаем следующие выражения:

$$
\begin{aligned}
& \mathbf{I}\left(P_{T}^{v} \mid Q_{T}^{v}\right)=\int_{0}^{T} \mathbf{E}_{P_{T}^{v}}\left[\frac{1}{2} \sigma_{1}^{2}\left(\beta_{t}^{v, *}\right)^{2}-\lambda\left(\ln Y_{t}^{v, *}-Y_{t}^{v, *}+1\right)\right] d t, \\
& \mathbf{I}\left(Q_{T}^{v} \mid P_{T}^{v}\right)=\int_{0}^{T} \mathbf{E}_{Q_{T}^{v}}\left[\frac{1}{2} \sigma_{1}^{2}\left(\beta_{t}^{v, *}\right)^{2}+\lambda\left(Y_{t}^{v, *} \ln Y_{t}^{v, *}-Y_{t}^{v, *}+1\right)\right] d t, \\
& \mathbf{H}^{(q)}(v)=\mathbf{E}_{R_{T}^{v}} \exp \left\{\int _ { 0 } ^ { T } \left(\frac { 1 } { 2 } \left(q(1-q)\left(\beta_{t}^{v, *}\right)^{2}\right.\right.\right. \\
&\left.\left.-\lambda\left(\left(Y_{t}^{v, *}\right)^{q}-q Y_{t}^{v, *}+q-1\right)\right) d t\right\} .
\end{aligned}
$$

Д о к а з а т е л ь с т в о. Выражения для информационных количеств получаются из формул, приведенных в предложениях 1, 2, 3 разд. 2.

Наконец, чтобы получить значение максимальной ожидаемой полезности, применим теорему 2 разд. 2, взяв закон распределения $\alpha$ равным $\mathscr{N}\left(0, T^{\prime}\right)$. 


\section{СПИСОК ЛИТЕРАТУРЫ}

1. Amendinger J., Becherer D., Schweizer M. A monetary value for initial information in portfolio optimization. - Finance Stoch., 2003, v. 7, № 1, p. 29-46.

2. Bertoin J. Lévy Processes. Cambridge: Cambridge Univ. Press, 1996, 265 p.

3. Bielecki T. R., Jeanblanc M. Indifference pricing of defaultable claims. In «Indifference Pricing: Theory and Applications», ed. by Carmona R. Princeton: Princeton Univ. Press, 2009, p. 211-240.

4. Biagini S., Frittelli M., Grasselli M. Indifference price with general semimartingales. - Math. Finance, 2011, v. 21, № 3, p. 423-446.

5. Carmona R. Indifference Pricing: Theory and Applications. Princeton: Princeton Univ. Press, 2009, 414 p.

6. Cawston S., Vostrikova L. An f-divergence approach for optimal portfolios in exponential Lévy models. In «Inspired by Finance: The Musiela Festschrift», ed. by Kabanov Yu. et al. Cham: Springer, 2014, p. 83-101.

7. Cawston S., Vostrikova L. Lévy preservation and associated properties for the $f$-divergence minimal equivalent martingale measures. In «Prokhorov and Contemporary Probability Theory», ed. by Shiryaev A. N. et al. Berlin: Springer, 2013, p. 163-196.

8. Eberlein E. Jump-type Levy Processes. In «Handbook of Financial Series». Berlin: Springer-Verlag, 2007, p. 439-455.

9. Eberlein E., Keller U. Hyperbolic distributions in finance. - Bernoulli, 1995, v. 1, № 3, p. 281-299.

10. Ellanskaya A., Vostrikova L. Utility maximization and utility indifference price for exponential semimartingale models and Hara utilities. - Труды МИАН, 2014, т. 287, c. $75-103$.

11. Goll T., Ruschendorf L. Minimax and minimal distance martingale measures and their relationship to portfolio optimisation. - Finance Stoch., 2001, v. 5, № 4, p. 557-581.

12. Gasbarra D., Valkeila E., Vostrikova L. Enlargement of filtration and additional information in pricing models: Bayesian approach. In «From Stochastic Calculus to Mathematical Finance», ed. by Kabanov Yu., et al. Berlin: Springer-Verlag, 2006, p. $257-285$.

13. Henderson $V$., Hobson D. Utility indifference pricing - an overview. In «Indifference Pricing: Theory and Applications», ed. by Carmona R. Princeton: Princeton Univ. Press, 209, p. 44-74.

14. Jacod J. Grossissement initiale, hypothèse ( $\left.\mathrm{H}^{\prime}\right)$ et théorème de Girsanov. In «Grossissements de filtrations: exemples et applications», ed. by Jeulin T., Yor M., et al. Berlin: Springer, 1980.

15. Jacod J. Calcul Stochastique et problèmes de Martingales. Berlin: Springer, 1979.

16. Жакод Ж., Ширяев А. Н. Предельные теоремы для случайных процессов. Т. 1, 2. М.: Физматлит, 1994.

17. Musiela M., Zariphopoulou T. An example of indifference prices under exponential preferences. - Finance Stoch., 2004, v. 8, № 2, p. 229-239.

18. Musiela M., Zariphopoulou T. Indifference prices and related measures. The University of Texas at Austin, Technical report, 2001. http://w.w.w.ma.utexas.edu/users/ zariphop/.

19. Sato K. Lévy Processes and Infinitely Divisible Distributions. Cambridge: Cambridge Univ. Press, 1999, 486 p.

20. Stricker C., Yor M. Calcul stochastique dèpendant d'un paramétre. - Warsch. ver. Geb., 1978, v. 45, p. 109-133. 